\title{
Kommunalwahlen unter veränderten Wettbewerbsbedingungen
}

\author{
Hiltrud Naßmacher
}

Angesichts der Niederlagen der CDU bei den letzten Oberbürgermeisterwahlen in Karlsruhe, Stuttgart und Frankfurt wurde bereits ein Niedergang der CDU als Großstadtpartei konstatiert. Es folgten weitere Amtsverluste in Kiel und Leipzig, die allerdings weniger überraschend waren als jener in Wiesbaden. In der Tat amtierten Ende 2012 in Städten über 100.000 Einwohner doppelt so viele (Ober-)Bürgermeister der SPD wie der CDU, während bei den stärksten Fraktionen in den stark fragmentierten Stadträten nahezu Gleichstand herrschte. Offensichtlich erlaubt die Stärke der Parteien keine sichere Prognose für die Wahl des Verwaltungschefs. Unterscheidet sich das Wahlverhalten zu den Wahlen für die Spitzenpositionen in den Städten von dem der Wahlen zu den Mitgliedern der Stadträte? Welchen Einfluss haben die institutionellen Reformen unter anderem auf die Bedeutung der Parteien? Welche gemeinsamen Einflüsse sind für das Wahlverhalten vor Ort bestimmend? Diesen Fragen soll im Folgenden nachgegangen werden.

\section{Bisheriger Forschungsstand}

Das Wahlverhalten auf der kommunalen Ebene ist bisher wenig untersucht worden. „Kommunales Wahlverhalten gilt ,als blinder Fleck der Wahlforschung." 1 Analysefähige Individualdaten für langfristige Vergleiche liegen nicht vor, weil erhebliche Kosten anfallen und ein Interesse potentieller Auftraggeber (Medien, Parteien) an solchen Erhebungen fehlt. Die Grundlagen für eine langfristig angelegte Aggregatdatenanalyse zu schaffen ist sehr zeitaufwendig und nur für eine gut begründete Auswahl von Städten und Gebieten überhaupt zu leisten. Denn im Zeitverlauf hat es nicht nur Gebiets- und Verfassungsreformen gegeben, sondern auch vielfältige Veränderungen des kommunalen Wahlrechts (insbesondere bei Amts- und Wahlzeiten, der Wahlgänge, der möglichen Zahl von Stimmen sowie bei Sperrklauseln). Eine solche Analyse würde allerdings dazu beitragen, eine wichtige Forschungslücke zu schließen.

Dennoch gibt es seit Jahrzehnten Hypothesen zum örtlichen Wahlverhalten, die allerdings auf eine breiter angelegte empirische Prüfung warten. Die Differenzthese wurde bereits von Karl-Heinz Naßmacher $1981^{2}$ betont. Er ging davon aus, dass ebenenspezifische Aspekte von Bedeutung sind, die sich unter anderem durch die Wahlsysteme und die Stadt-/Gemeindegröße ergeben, die spezifische Milieus zur Folge haben. ${ }^{3}$ Dagegen prognostizierte

1 Thorsten Faas, Wahlbeteiligung, in: Jan W. van Deth / Markus Tausendpfund (Hrsg.), Politik im Kontext: Ist alle Politik lokale Politik? Individuelle und kontextuelle Determinanten politischer Orientierungen, Wiesbaden 2013, S. 413 - 440, S. 418 (unter Verweis auf Oscar W. Gabriel in Fn. 16).

2 Er hatte dies für die Kommunalwahlen in Niedersachsen 1972 und 1976 nachgewiesen. KarlHeinz Naßmacher, Empirische Dimensionen einer kommunalen Verwaltungsreform, in: Dietrich Thränhardt / Herbert Uppendahl (Hrsg.), Alternativen lokaler Demokratie, Königstein/Taunus 1981, S. 43 - 83, S. 47 f.

3 Vgl. Karl-Heinz Naßmacher, Parteien im Abstieg. Wiederbegründung und Niedergang der Bauernund Bürgerparteien in Niedersachsen, Wiesbaden 1989, S. 265. 
Paul Kevenhörstert schon Mitte der 1970er Jahre, dass sich das Wahlverhalten aller Ebenen angesichts der Dominanz ähnlicher Vermittlungsprozesse im Wahlkampf angleichen würde.

Aus theoretischer Sicht sollten es Impulse aus kleinräumigeren Kontexten sein, die einen Einfluss auf die Wahlbeteiligung haben. ${ }^{5}$ Aber die Beteiligung an Kommunalwahlen ist regelmäßig geringer als an Bundestagswahlen. ${ }^{6}$ Offensichtlich folgt letztere einer anderen Logik.7 Manfred Güllner ${ }^{8}$ sieht die Schuld bei der „politischen Klasse“, die sich nicht an den Bedürfnissen der Menschen vor Ort ausrichte. Hier stehen die Persönlichkeiten in herausgehobenen Positionen besonders im Fokus, seit der Direktwahl vor allem die (Ober-)Bürgermeister und die Führungspersönlichkeiten in den Fraktionen.

Die bisherigen Forschungsergebnisse wurden meist mit einer spezifischen Fragestellung (Bürgermeisterwahlen), nur für einzelne Bundesländer (Baden-Württemberg9 und Sachsen ${ }^{10}$ ), eine Wahl (1999 in Nordrhein-Westfalen nach der Kommunalwahlreform ${ }^{11}$ ) oder eine Stadtgröße (kreisfreie Städte in NRW12) erzielt. Auch Untersuchungen zum Wahlverhalten allgemein beschränken sich auf ein Bundesland oder eine Region (Nordrhein-Westfalen ${ }^{3}$, das Ruhrgebiet ${ }^{14}$ ). Bei breiter angelegter Forschungsstrategie wird eine begrenzte Zahl von Fallstudien für eine Wahl15 beziehungsweise nur für eine einzelne Stadt ${ }^{16}$ in die Analyse einbezogen. Die aktuellsten Ergebnisse basieren auf Bevölkerungsbefragungen in

4 Vgl. Paul Kevenhörster, Parallelen und Divergenzen zwischen gesamtsystemaren und kommunalem Wahlverhalten, in: Konrad Adenauer Stiftung (Hrsg.), Kommunales Wahlverhalten, Bonn 1976, S. $241-283$.

5 Vgl. Thorsten Faas, a.a.O. (Fn. 1), S. 418.

6 Vgl. Armin Schäfer, Alles halb so schlimm? Warum eine sinkende Wahlbeteiligung der Demokratie schadet, Max-Planck-Institut für Gesellschaftsforschung, Köln 2009, http://www.mpifg.de/aktuelles/themen/doks/jahrbuch-09-10-schaefer-280509.pdf (Abruf am 22. November 2013), S. 6.

7 Vgl. Thorsten Faas, a.a.O. (Fn. 1), S. 424.

8 Vgl. Manfred Güllner, Eine vergessene Ebene der Politik, in: Cicero vom 15. September 2011, http://www.cicero.de/berliner-republik/eine-vergessene-ebene-der-politik/43034/seite/3 (Abruf am 29. November 2011).

9 Vgl. Hans-Georg Wehling / H. Jörg Siewert, Die Bürgermeister in Baden-Württemberg. Eine Monographie, Stuttgart 1987; Bernhard Löfler / Walter Rogg, Kommunalwahlen und kommunales Wahlverhalten, in: Theodor Pfitzer / Hans-Georg Wehling (Hrsg.), Kommunalpolitik in BadenWürttemberg, Stuttgart 2000, S. $108-124$.

$10 \mathrm{Vgl}$. Manfred Scheer, Was ist los mit der Demokratie in Sachsens Rathäusern?, Leipzig 2003.

11 Vgl. Uwe Andersen / Rainer Bovermann (Hrsg.), Im Westen was Neues. Kommunalwahl 1999 in NRW, Opladen 2002.

12 Vgl. Hiltrud Naßmacher, Die Auswahl der Kandidaten muss sorgfältiger werden, in: dies., Baustelle Stadt. Effizienz und Bürgernähe ohne Demokratie und Nachhaltigkeit?, Wiesbaden 2006, S. 22 -34 .

13 Vgl. Rainer Bovermann, Kommunalwahlen und kommunales Wahlverhalten, in: Uwe Andersen (Hrsg.), Kommunalpolitik in Nordrhein-Westfalen im Umbruch, Köln 1998, S. 160 - 183.

14 Vgl. Rainer Bovermann, Wahlen im Ruhrgebiet in vergleichender Perspektive, in: ders. / Stefan Goch / Heinz-Jürgen Priamus (Hrsg.), Das Ruhrgebiet - ein starkes Stück Nordrhein-Westfalen. Politik in der Region 1946-1996, Essen 1996, S. 336 - 364.

15 Vgl. Rainer Bovermann, Kommunales Wahlverhalten zwischen Partei-, Themen- und Kandidatenorientierung, in: Uwe Andersen / ders., a.a.O. (Fn. 11), S. 115 - 159; dies., Einführung: Die Uraufführung der Bürgermeisterdirektwahl in NRW, in: ebenda, S. 7 - 35, S. 7 - 18, S. 8.

16 Vgl. Oscar W. Gabriel, Kommunales Wahlverhalten. Parteien, Themen und Kandidaten, in: ders. / Frank Brettschneider / Angelika Vetter (Hrsg.), Politische Kultur und Wahlverhalten in einer Großstadt, Opladen 1997, S. 147 - 168. 
einer zufälligen Auswahl von 26 Städten und Gemeinden mit 5.000 bis 50.000 Einwohnern in Hessen aus dem Jahre 2008/2009 und zwei Großstädten (Kassel und Wiesbaden). ${ }^{17}$

Das Wahlverhalten auf der örtlichen Ebene könnte nach den bisherigen Hypothesen dadurch beeinflusst sein, dass

- es spezifische Wettbewerbsregeln (Wahlsystem) gibt,

- die von Lokalparteien mitgestalteten örtlichen Milieus eine besondere Rolle spielen,

- die Vermittlungsprozesse vor Ort sich von bundesweiten Wahlen unterscheiden,

- auf der kommunalen Ebene den Kandidaten eine größere Bedeutung zukommt oder deren Verhalten in bestimmten Sachfragen.

Die folgende Analyse kann sich vor diesem Hintergrund auf die Auswertung einer im Oktober 2012 veröffentlichten umfassenden Datensammlung zu den aktuellen Kommunalwahlergebnissen aus allen Bundesländern stützen. ${ }^{18}$ Dabei sind die neuesten Kommunalwahlen am wichtigsten, da sie bereits die veränderten Wettbewerbsbedingungen berücksichtigen. Die eigenen Berechnungen beschränken sich auf die Städte mit über 50.000 Einwohnern, und zwar 123 unter 100.000 und 77 Städte mit über 100.000 Einwohnern. ${ }^{19}$

\section{Wablen der Mitglieder des Rates}

Offenbar scheinen Wahlberechtigte den Kommunalwahlen genauso wie den Landtagswahlen und den Wahlen zum Europäischen Parlament nicht die gleiche Bedeutung beizumessen wie den Bundestagswahlen, so dass sie auch als Nebenwahlen bezeichnet werden können. Ein Indikator dafür ist die Wahlbeteiligung.

\subsection{Beteiligung an den Stadtratswahlen}

Bei längerfristiger Betrachtung ist die Wahlbeteiligung stark gesunken, zum Beispiel in Rheinland-Pfalz von 1989 bis 2009 um 22 Prozentpunkte, in Brandenburg von 1998 bis 2003 um 32, in Sachsen-Anhalt von 1994 bis 2007 um 30 und in Thüringen von 1994 bis 2009 um 19 Prozentpunkte. Gingen in Mecklenburg-Vorpommern 2011 nur noch die Hälfte der Wahlberechtigten zur Wahl, so waren es 1990 noch etwa 70 Prozent. ${ }^{20}$

Zuweilen finden Kommunalwahlen in unmittelbarer zeitlicher Nähe zu Bundestagswahlen statt. Dies war zum Beispiel im Superwahljahr 2009 in acht Bundesländern der Fall. Die Beteiligung an den Kommunalwahlen müsste dann höher sein, denn es ist anzunehmen,

17 Vgl. Jan W. van Deth / Markus Tausendpfund, Einführung: Ist alle Politik lokale Politik?, in: dies. (Hrsg.), a.a.O. (Fn. 1), S. $9-31$, S. 17 ff.

18 Vgl. Konrad Adenauer Stiftung (Hrsg.), Kommunales Wahllexikon, St. Augustin 2012.

19 Vgl. ebenda, S. 8 - 26, S. 27 - 52.

20 Vgl. Landeswahlleiterin Nordrhein-Westfalen, http://www.wahlergebnisse.nrw.de/kommunalwahlen/2009/index.html (Abruf am 29. November 2012); Statistisches Amt Mecklenburg-Vorpommern, http://www.statistik-mv.de/ (Abruf am 13. Dezember 2012); Landeswahlleiter RheinlandPfalz, Schnellauswertung der Kommunalwahlen 2009 in Rheinland-Pfalz, Mainz 2009; Landesamt für Zentrale Dienste - Statistisches Amt, Gemeinderatswahlen 1946 bis 2009 im Saarland, Saarbrücken 2009; Thüringer Landesamt für Statistik, Kommunalwahlen in Thüringen am 7. Juni 2009, Endgültige Ergebnisse, Erfurt 2009, S. 10. 


\begin{tabular}{|c|c|c|c|}
\hline \multicolumn{4}{|c|}{$\begin{array}{l}\text { Tabelle 1: Wablbeteiligung bei unmittelbarer Nähe von Kommunal- und Bundestagswahlen } \\
\text { (in Prozent) }\end{array}$} \\
\hline Land & Wahlen & Wahljahre & Wahlbeteiligung \\
\hline \multirow{2}{*}{ Baden-Württemberg } & ohne BTW & 2004 & 52,5 \\
\hline & auch BTW & 2009 & 51,0 \\
\hline \multirow{2}{*}{ Mecklenburg-Vorpommern } & ohne BTW & 2004 & 44,9 \\
\hline & auch BTW & 2009 & 46,6 \\
\hline \multirow{2}{*}{ Rheinland-Pfalz } & ohne BTW & 2004 & 57,8 \\
\hline & auch BTW & 2009 & 55,2 \\
\hline \multirow{2}{*}{ Saarland } & ohne BTW & 2004 & 56,3 \\
\hline & auch BTW & 2009 & 57,5 \\
\hline \multirow{2}{*}{ Sachsen } & ohne BTW & 2004 & 46,6 \\
\hline & auch BTW & 2009 & 47,5 \\
\hline \multirow{2}{*}{ Sachsen-Anhalt } & ohne BTW & 2004 & 42,1 \\
\hline & auch BTW & 2009 & 37,8 \\
\hline \multirow{2}{*}{ Thüringen } & ohne BTW & 2004 & 50,6 \\
\hline & auch BTW & 2009 & 53,2 \\
\hline \multirow{2}{*}{ Nordrhein-Westfalen } & ohne BTW & 2004 & 54,4 \\
\hline & auch BTW & 2009 & 52,4 \\
\hline
\end{tabular}

dass die anlaufenden Mobilisierungsaktivitäten für die bevorstehende Bundestagswahl auf die kommunale Ebene ausstrahlen beziehungsweise diejenigen Wähler, die sich an der Bundestagswahl beteiligen, auch ihre Stimme für die Wahl des Rates abgeben. Die Zahlen zeigen dies in vier ostdeutschen Bundesländern und im Saarland, nicht allerdings in SachsenAnhalt, Baden-Württemberg, Rheinland-Pfalz und Nordrhein-Westfalen (vgl. Tabelle 1).

\subsection{Personelles Angebot für die Stadträte}

„Die Weigerung so vieler Bürger, sich an Wahlen vor Ort zu beteiligen, dürfte in erster Linie die Reaktion auf das vielerorts als Zumutung empfundene personale ... Angebot der Parteien sein." 21 Bevor darüber der Stab gebrochen wird, sollten sich die Kritiker überlegen, wer neben seiner Berufstätigkeit noch ein Kommunalmandat ausfüllen kann, das schon in einer mittleren Großstadt in den 1980er Jahren hinsichtlich der zeitlichen Belastung einer Halbtagstätigkeit entsprach. ${ }^{22}$ Die dort erhobenen Werte dürften sich nicht wesentlich verändert haben. Hier engt sich der Kreis der Aktiven schon sehr auf diejenigen ein, die in ihren Beru-

21 Manfred Güllner, a.a.O. (Fn. 8), S. 1.

22 Vgl. Hiltrud Naßmacher / Karl-Heinz Naßmacher, Kommunalpolitik in Deutschland, Opladen 1999, S. 277; genauere Angaben aus verschiedenen empirischen Untersuchungen in: dies., 2. Auflage, Opladen 2007, S. 212. Diese Zeitangaben wurden im Wesentlichten durch die Erhebungen aus dem Jahre 2003 bestätigt. Lars Holtkamp, Professionalisierung der Kommunalpolitik, in: Michael Edinger / Werner J. Patzelt (Hrsg.), Politik als Beruf, PVS-Sonderheft 44, Wiesbaden 2011, S. $103-120$, S. $108 \mathrm{ff}$. 
fen flexibel oder durch Verbeamtung abgesichert sind (nachgeordnete Mitarbeiter aus Verwaltungen, Verbänden und Lehrer), die ein (Klein-) Unternehmen führen, in dem der Chef auch einmal vertreten werden kann (zum Beispiel in Apotheken oder Handelsunternehmen). Hinzu kommen Hausfrauen, deren Kinder aus dem Haus sind und Rentner. ${ }^{23}$ Hans-Georg Wehling spricht für Baden-Württemberg von Parlamenten, in denen Honoratioren dominieren. ${ }^{24}$ Insbesondere in der Umbruchphase der neuen Bundesländer zeigte sich angesichts der wirtschaftlichen Unsicherheit, aber auch zuweilen aus politischer Enttäuschung eine starke Fluktuation. ${ }^{25}$ In kleineren Städten und Gemeinden mussten vor allem die kleinen bürgerlichen Parteien häufig örtlich bekannte Nichtparteimitglieder nominieren, um breitere Zustimmung zu mobilisieren und dadurch ihre lokalen Milieus zu stabilisieren ${ }^{26}$, eine Strategie, die bei den Freien Wählern in Baden-Württemberg immer schon erfolgreich war. ${ }^{27}$ Den großen Parteien scheint erst allmählich klar zu werden, dass aus der geringer werdenden Zahl von Parteiaktivisten nicht genügend qualifizierte Kandidaten zu rekrutieren sind.

Junge Leute (Berufsanfänger und Studenten) können zwar viel über Kommunalpolitik durch die Tätigkeit als Mandatsträger lernen und dabei Kontakte knüpfen, die ihnen unter Umständen sogar einen Berufseinstieg ermöglichen; als Sprungbrett in politiknahe berufliche Positionen (Mitarbeiter bei der Lokalpresse oder bei Politikern) ist die Mitarbeit in der Kommunalpolitik nämlich immer noch geeignet. Die Freude an der Tätigkeit muss aber schon groß sein, denn finanziell lohnt sich das Engagement nicht. Dies gilt besonders für kleinere Gemeinden. Selbst in der Stadt Oldenburg mit 160.000 Einwohnern gibt es 266 Euro monatlich und für die Teilnahme an Sitzungen jeweils 24 Euro, in den kleinen Gemeinden der Landkreise teilweise nur 30 Euro und für die Teilnahme an Sitzungen zum Teil nur 15 Euro. ${ }^{28}$

Ein wichtiger Anreiz, sich die zusätzliche Belastung aufzuerlegen, ist die Chance auf ein höherwertiges Mandat (in den Parlamenten von Land, Bund oder Europa), denn nach wie vor gilt die kommunalpolitische Tätigkeit als eine Qualifikationsvoraussetzung. ${ }^{29}$ Allerdings

23 Vgl. Hiltrud Naßmacher, a.a.O. (Fn. 12), S. 64.

24 Vgl. Hans-Georg Wehling, Auswirkungen der Kommunalverfassungen auf das lokale politische Handeln. Erfahrungen mit dem baden-Württembergischen Modell, in: Dieter Schimanke (Hrsg.), Stadtdirektor oder Bürgermeister, Basel 1989, S. 84 - 96, S. 89.

25 Vgl. Renate Hürtgen / Henry Kreikenbom / Bärbel Möller / Peter Müller / Bernd Schaarschmidt / Petra Weigel, Sozialpolitische Interessenvermittlungsstrukturen im Transformationsprozess in den regionalen Zentren Frankfurt (Oder) und Jena, in: Hiltrud Naßmacher / Oskar Niedermayer I Hellmut Wollmann (Hrsg.), Politische Strukturen im Umbruch, Berlin 1994, S. 17 - 118, S. 41; Kathrin Susann Becher, Mandatsniederlegungen auf kommunaler Ebene, Opladen 1997, S. 244.

26 Zur Bedeutung der lokalen Verortung der Parteien vgl. Karl-Heinz Naßmacher, a.a.O. (Fn. 3), S. 180 ff., S. 265.

27 Vgl. Herbert Schneider, Kreispolitik im ländlichen Raum, München 1985, S. 99; für Niedersachsen: Karl-Heinz Naßmacher / Wolfgang Rudzio, Das lokale Parteiensystem auf dem Lande. Dargestellt am Beispiel der Rekrutierung von Gemeinderäten, in: Hans-Georg Wehling (Hrsg.), Dorfpolitik, Opladen 1978, S. 127 - 142, S. 132 ff.

28 Vgl. Nord-West-Zeitung vom 23. Dezember 2011; weiter quantitative Informationen dazu bei Lars Holtkamp, a.a.O. (Fn. 22), S. 108; Marion Reiser, Ressourcen oder mitgliederbasiert? Zwei Formen der Professionalisierung auf der lokalen Ebene und ihre institutionelle Ursachen, in: Michael Edinger / Werner J. Patzelt, a.a.O. (Fn. 22), S. 121 - 144, S. 131.

$29 \mathrm{Vgl}$. Jens Borchert, Drei Welten politischer Karrieremuster in Mehrebenensystemen: die USA, Deutschland und Brasilien im Vergleich, in: Michael Edinger / Werner J. Patzelt, a.a.O. (Fn. 22), S. $273-296$, S. 284. 
ist der Verbleib von jungen Leuten in der Mandatstätigkeit häufig nicht von Dauer, weil Berufseinstieg und Studium große Bereitschaft zur Mobilität fordern. Höherwertige Mandate sind keine sichere Option und derjenige, der in jungen Jahren ein solches Mandat erringt, schafft häufig nicht den Aufstieg in der Hierarchie und hat bei Abwahl selten eine Wiedereinstiegsmöglichkeit in seinen (vorher nicht ausgeübten) Beruf. 30

Durch das berufsbedingte Ausscheiden vieler Mandatsträger, aber auch durch ein Verbleiben von neuen Mitgliedern in nachgeordneten Positionen (häufig mindestens eine Wahlperiode lang) kommt es zum Personalstau in den Fraktionen, also zur Dominanz der schon einige Wahlperioden Agierenden. Für die Fraktionen selbst ergibt sich dadurch eine wachsende Professionalisierung der Führungspersönlichkeiten, bei den Parteimitgliedern und externen Beobachtern aber eher Frustration über den Mangel an innerparteilicher Demokratie. Die Wahlberechtigten beurteilen die jahrelange Dominanz einzelner Repräsentanten eher negativ als Parteienfilz, und gleichzeitig leidet die Qualität der Fraktionen häufig durch zufällige Nachrücker, die eigentlich gar kein Mandat angestrebt hatten.

Diejenigen Wahlberechtigten, die während der Wahlperiode Sachentscheidungen zur Bearbeitung öffentlicher Aufgaben kritisch begleiten und dabei Debatten über Prioritätensetzungen nur als Profilierung der Parteien oder ihrer Führungspersönlichkeiten empfinden, werden damit eher unzufrieden sein, sich von der Kommunalpolitik abwenden und sich an Kommunalwahlen nicht mehr beteiligen. Die Zufriedenheit mit der Leistung der Mandatsträger ist individuell verschieden und abhängig davon, wie stark als Bewertungskriterium die eigenen Interessen im Vordergrund stehen oder die Bedeutung für die Gesamtgemeinde. ${ }^{31}$ Die Kommunikation im sozialen Umfeld ist hier ebenfalls von Bedeutung. ${ }^{32}$

\subsection{Wirkungen unterschiedlicher Wahlgesetze für die Stadtratswahlen}

Bei der Kandidatenaufstellung für Kommunalwahlen spielen trotz der geringer werdenden Auswahlmöglichkeiten für Personalvorschläge die innerparteilichen Machtstrukturen natürlich eine wichtige Rolle. Jedenfalls wurde häufig das quasi existierende Monopol der Parteien (und damit das der Führungspersönlichkeiten) bei der Kandidatenaufstellung als Problem gesehen. 33

Durch Reformen der Kommunalwahlgesetze sollte den Wahlberechtigten ein Mitspracherecht bei der Reihenfolge der Parteivorschläge auf den Listen eingeräumt werden; dies ist in Baden-Württemberg, Bayern und Rheinland-Pfalz besonders ausgeprägt. Hier hat der Wahlberechtigte so viele Stimmen, wie Mandate für das Kommunalparlament zu vergeben sind. Schleswig-Holstein und Hessen sind inzwischen gefolgt. In Bremerhaven hat der Wähler seit den letzten Kommunalwahlen fünf Stimmen, in Niedersachsen und den neuen Bundesländern sind es drei. Dabei ist Kumulieren und Panaschieren mit jeweils drei Stimmen

$30 \mathrm{Vgl}$. Maria Kreiner, Mandat auf Zeit. Eine Verbleibsstudie über ehemalige Bundestagsabgeordnete, Baden-Baden 2006, S. 86 - 90, S. 134 ff.

31 Vgl. Sebastian Kuhn, Lokale Orientierungen, in: Jan W. van Deth / Markus Tausendpfund, a.a.O. (Fn. 1), S. 35 - 65, S. 37, S. 45.

32 Vgl. Sigrid Roßteutscher / Philipp Scherer, Wertorientierungen, in: Jan W. van Deth / Markus Tausendpfund, a.a.O. (Fn. 1), S. $67-91$, S. 88.

33 Eigene Beobachtungen aus der kommunalpolitischen Praxis in Solingen und Oldenburg sowie Fachgespräche mit Klaus Simon, Universität Konstanz. 
möglich. Nur in Nordrhein-Westfalen und dem Saarland haben Wahlberechtigte bisher lediglich eine Stimme.

Mehrstimmenwahlen bewirken, dass diejenigen, die bei vorangegangen Wahlen besonders viele Stimmen geholt haben, glauben, ihren Parteien und Wählergruppierungen einen guten Dienst zu tun, wenn sie immer wieder kandidieren. In Baden-Württemberg haben sich allerdings vorausschauende Fraktionsvorsitzende bei der Listenzusammensetzung durchaus um neues Personal auf den vorderen Plätzen bemüht (junge Frauen, Berufsgruppen, die bisher nicht vertreten waren, junge Aktivisten), weil bekannt ist, dass diese Kandidaten dann die besseren Chancen haben, gewählt zu werden. ${ }^{34}$ Schon bei Städten mittlerer Größe zeigt sich, dass die wenigsten Kandidaten persönlich bekannt sind. 35 In Rheinland-Pfalz wurde in kleineren Gemeinden und Städten 2004 am häufigsten von den Möglichkeiten des Kumulierens und Panaschierens Gebrauch gemacht. ${ }^{36}$ So betont Norbert Kersting 37 , dass sich die Abstimmungen mit vielen Stimmen am ehesten für kleinere Gemeinden eignen.

Generell konnte der Rückgang der Wahlbeteiligung durch die Wahlrechtsänderungen kaum aufgehalten werden; dies gilt zum Beispiel in Schleswig-Holstein und Hessen, obwohl in Schleswig-Holstein 95 Prozent der Kommunen weniger als 10.000 Einwohner haben, im Gegensatz zu Hessen mit nur 61 Prozent der Gemeinden in dieser Größenordnung. Es zeigt sich aber, dass die Bundesländer mit sehr vielen kleinen Gemeinden (Einwohnerzahl unter 10.000), in denen das komplizierte Wahlrecht schon tradiert ist, eine höhere Wahlbeteiligung bei mehr Stimmen haben. Dies ist bei Baden-Württemberg, Bayern und RheinlandPfalz mit 77,8 Prozent, 89,6 beziehungsweise 98,0 Prozent in kleinen Gemeinden 38 der Fall (vgl. Tabelle 2) und deutet auf einen Gewöhnungsprozess hin. Allerdings ist das Bild keineswegs einheitlich. Landesinterne und gemeindespezifische Besonderheiten mögen die Ursachen sein. Aber vor allem die individuellen Ressourcen gelten für die Wahlbeteiligung (wie für andere Beteiligungsformen auch ${ }^{39}$ ) als wichtiger denn die Gemeindegröße.

\subsection{Folgen des Wegfalls der Fünf-Prozent-Klausel}

Durch die Beseitigung der Fünf-Prozent-Klausel als Qualifikationshürde haben sehr viel mehr Kandidatenangebote Chancen, bei den Wahlen Mandate zu gewinnen. Dies ging in

34 Vgl. Hiltrud Naßmacher, a.a.O. (Fn. 12), S. 65.

35 Diesem Problem versuchen Parteien in Baden-Württemberg schon seit Jahrzehnten dadurch zu begegnen, dass sie bekannte Persönlichkeiten, unter anderem aus Parteien und Verbänden, für ihre Listen zu gewinnen suchen. Vgl. dies., Parteien und Wählergruppen in der Kommunalpolitik, in: Oscar W. Gabriel / Oskar Niedermayer / Richard Stöss (Hrsg.), Parteiendemokratie in Deutschland, Bonn 1997, S. 427 - 442, S. 432.

36 Der Landeswahlleiter Rheinland-Pfalz, Kommunalwahlen in Rheinland-Pfalz am 13. Juni 2004 - Auswertung des Wählerverhaltens, Mainz 2004, http://www.wahlen.rlp.de (Abruf am 23. Februar 2013), S. 5.

37 Vgl. Norbert Kersting, Wahlbeteiligung auf historischem Tiefstand, in: FAZ vom 27. März 2011, http://www.faz.net/akutell/rhein-main/hessen/kommunalwahlen-wahlbeteiligung-auf-historischem-tiefstand-1607333.html (Abruf am 19. September 2012).

38 Gemeindegrößen nach Statistisches Bundesamt (Hrsg.), Statistisches Jahrbuch 2011, Wiesbaden 2011, S. $40 \mathrm{f}$.

39 Vgl. Oscar W. Gabriel, Politische Partizipation, in: Jan W. van Deth / Markus Tausendpfund, a.a.O. (Fn. 1), S. $381-411$, S. 407. 


\begin{tabular}{|c|c|c|c|}
\hline Kommunalwahlgesetz & Bundesland & Wahljahre & Wahlbeteiligung \\
\hline \multirow{10}{*}{$\begin{array}{l}\text { Anzahl der Stimmen } \\
=\text { zu vergebende Mandate }\end{array}$} & \multirow{2}{*}{ Baden-Württemberg } & 1999 & 53,0 \\
\hline & & 2004 & 52,0 \\
\hline & \multirow{2}{*}{ Bayern } & 2002 & 63,2 \\
\hline & & 2008 & 59,5 \\
\hline & \multirow{2}{*}{ Hessen } & 2006 & 45,8 \\
\hline & & 2011 & 47,7 \\
\hline & \multirow{2}{*}{ Rheinland-Pfalz } & 1999 & 62,9 \\
\hline & & 2004 & 57,8 \\
\hline & \multirow{2}{*}{ Schleswig-Holstein } & 2003 & 54,5 \\
\hline & & 2008 & 49,5 \\
\hline \multirow{12}{*}{ drei Stimmen } & \multirow{2}{*}{ Niedersachsen } & 2006 & 53,4 \\
\hline & & 2011 & 47,7 \\
\hline & \multirow{2}{*}{ Brandenburg } & 2003 & 45,8 \\
\hline & & 2008 & 49,4 \\
\hline & \multirow{2}{*}{ Sachsen } & 1999 & 49,6 \\
\hline & & 2004 & 46,0 \\
\hline & \multirow{2}{*}{ Mecklenburg-Vorpommern } & 2004 & 44,9 \\
\hline & & 2011 & 51,1 \\
\hline & \multirow{2}{*}{ Sachsen-Anhalt } & 2004 & 43,9 \\
\hline & & 2007 & 36,4 \\
\hline & \multirow{2}{*}{ Thüringen } & 1999 & 50,2 \\
\hline & & 2004 & 42,2 \\
\hline \multirow{4}{*}{ eine Stimme } & \multirow{2}{*}{ Nordrhein-Westfalen } & 1999 & 58,1 \\
\hline & & 2004 & 54,4 \\
\hline & \multirow{2}{*}{ Saarland } & 1999 & 59,2 \\
\hline & & 2004 & 56,3 \\
\hline
\end{tabular}

Nordrhein-Westfalen zu Lasten der großen Parteien CDU und SPD, deren Verlust in der Hälfte der Fälle im zweistelligen Bereich lag und mit 18 Prozentpunkten (Wuppertal) am höchsten war. ${ }^{40}$ Dagegen haben die kleinen Parteien, die auch auf Bundes- und Landesebene kandidieren, bessere Chancen, Mandate zu gewinnen: die Linken und neuerdings auch die Piraten, die erstmalig 2011 in Niedersachen und Hessen bei den Kommunalwahlen antraten und ohne den Wegfall der Fünf-Prozent-Klausel keine Mandate gewonnen hätten (vgl. Tabelle 3).

Es zeigt sich, dass in den großen Städten in Niedersachsen und Hessen überwiegend die Wahlbeteiligung höher lag als in den vorangegangenen Wahlen. In Niedersachsen konnten die Piraten aber bei sinkender Wahlbeteiligung in Göttingen und Hannover von anderen Parteien Stimmen gewinnen sowie bei höherer Wahlbeteiligung in Hildesheim, Lüneburg, Osnabrück und Wolfenbüttel mehr als den Zuwachs an abgegebenen Stimmen auf sich

40 Für die kreisfreien Städte in Nordrhein-Westfalen im Vergleich der Wahlen 1999 und 2004 vgl. Tabelle 2, in: Hiltrud Naßmacher, a.a.O. (Fn. 12), S. 29. 


\begin{tabular}{|l|c|c|c|c|}
\hline $\begin{array}{l}\text { Tabelle 3: Ausschöpfen weiterer Wählerpotentiale, hier der Piraten 2011 (in Prozent, } \\
\text { Differenz in Prozentpunkten) }\end{array}$ \\
\hline & Piraten & $\begin{array}{c}\text { Wahlbeteiligung } \\
2006\end{array}$ & $\begin{array}{c}\text { Wahlbeteiligung } \\
2011\end{array}$ & Differenz \\
\hline Niedersachsen & & & & \\
Braunschweig & 3,9 & 49,3 & 49,5 & $+0,2$ \\
Göttingen & 3,7 & 50,4 & 49,8 & $-0,6$ \\
Hannover & 3,5 & 47,1 & 44,6 & $-2,5$ \\
Hildesheim & 2,9 & 52,3 & 53,8 & $+1,5$ \\
Lüneburg & 4,1 & 52,9 & 54,3 & $+1,4$ \\
Oldenburg & 2,8 & 44,4 & 47,4 & $+3,0$ \\
Osnabrück & 2,0 & 47,1 & 47,6 & $+0,5$ \\
Wilhelmshaven & 1,7 & 41,0 & 50,8 & $+9,8$ \\
Wolfsburg & 3,9 & 46,1 & 49,8 & $+3,7$ \\
Wolfenbüttel & 3,5 & 55,8 & 57,2 & $+1,4$ \\
Land Niedersachsen & 1,0 & 51,7 & 52,2 & $+1,8$ \\
\hline Hessen & & & & \\
Darmstadt & 2,9 & 43,7 & 49,4 & $+5,7$ \\
Frankfurt & 2,0 & 40,4 & 42,2 & $+1,8$ \\
Gießen & 2,8 & 44,8 & 48,0 & $+3,2$ \\
Kassel & 2,7 & 37,1 & 42,2 & $+5,1$ \\
Marburg & 1,9 & 43,9 & 49,0 & $+5,1$ \\
Wiesbaden & 2,1 & 39,6 & 41,4 & $+1,8$ \\
Land Hessen & 1,3 & 45,8 & 47,7 & $+1,9$ \\
\hline Quelle: Daten des Landesbetriebs für Statistik und Kommunikationstechnologie, http://www.nls.nieder- \\
sachsen.de; Daten des Hessischen Statistischen Landesamtes, http://www.statistik-hessen.de (eigene Zu- \\
sammenstellung). & & & \\
\hline
\end{tabular}

vereinigen. Wilhelmshaven bildet die Ausnahme. In Hessen konnten die Piraten in keinem Fall den Zuwachs an Wählern für sich verbuchen, mit einer geringen Abweichung in Wiesbaden. Die Frage, ob durch den Wegfall der Fünf-Prozent-Klausel neue Wähler an die Wahlurne gebracht wurden und ob dies auch durch die neue Partei bewirkt wurde, lässt sich wohl erst bei Untersuchungen zu weiteren Wahlen beantworten.

Weiterhin sind auch bisher nur regional bedeutende Parteien erfolgreich, besonders die Freien Wähler. Die höchsten Stimmenzahlen erreichen sie allerdings in ihren „Stammländern" Baden-Württemberg und Bayern: Sindelfingen (21,7 Prozent), Ludwigsburg (17,8 Prozent), Baden-Baden (17,3 Prozent) und Ingolstadt (15,2 Prozent). Daneben gibt es ein weiteres vielfältiges Kandidatenangebot aus Gruppierungen, die nicht leicht auf der RechtsLinks-Skala einzuordnen sind und nur auf der kommunalen Ebene kandidieren. Wie aus eigenen langjährigen Beobachtungen und älteren Untersuchungen bekannt, lehnen sie eine solche Festlegung ab, weil sie nur für das kommunale Gemeinwohl arbeiten wollen. ${ }^{41}$ Sie glauben, sich aus bundes- und landespolitischen Parteikonflikten heraushalten zu können.

41 Vgl. Marion Reiser / Adrienne Krappidel, Parteien ohne Parteilichkeit? Analysen zum Profil parteifreier Gruppierungen, in: SFB 580 Mitteilung, Projekt Kommunale Wählergemeinschaften, H. 25/2008, S. $74-97$, S. 85. 
Dabei verkennen sie, dass es auch auf der kommunalen Ebene um grundlegende gesellschaftliche Konflikte geht, die denen auf der Bundes- und Landesebene entsprechen, zum Beispiel bei der Kinderbetreuung, bei Schulformen, beim Ausbau von Straßen oder dem öffentlichen Verkehrsangebot. Wählergruppen setzen mehr als die Parteien auf Persönlichkeiten, während die überörtlich aktiven Parteien immer noch auf ihren „Markenkern“ zurückgreifen können, wenn er auch auf der kommunalen Ebene stärker verblasst. In Gemeinden mit Beschäftigungsproblemen tendieren die Wähler zu linken Positionen ${ }^{42}$, in Gemeinden mit vielen Haushalten mit hohen Einkommen sind postmaterialistische Positionen erkennbar. 43

Haben mehr Listen mit Kandidatenvorschlägen und mit Aussicht auf Erfolg auch generell dazu geführt, dass die Wahlbeteiligung besonders hoch war? In Städten über 100.000 Einwohner, in denen 2009 eine höhere Wahlbeteiligung als im Landesdurchschnitt erzielt wurde, konnten sieben Parteien und sonstige Gruppierungen Mandate im Rat erringen, mit Ausnahme von Bergisch-Gladbach mit nur sechs und Münster mit acht Gruppierungen im Rat. In Nordrhein-Westfalen kam zudem noch der Wechsel vom Stimmenverrechnungsverfahren d'Hondt zum Verfahren Hare-Niemeyer für kleinere Gruppierungen begünstigend bei der Mandatsvergabe hinzu. ${ }^{44}$ Bei den niedrigsten Wahlbeteiligungen 2009 ist die Ausdifferenzierung der Räte aber genauso hoch mit sieben bis neun unterschiedlichen Parteien und Gruppierungen. Werden die Städte mit unter 100.000 bis 50.000 Einwohner einbezogen, so geht hohe Wahlbeteiligung 2009 auch zuweilen mit geringerer Ausdifferenzierung des Rates einher, zum Beispiel in Erftstadt, Rheine und Willich mit nur vier und Hürth mit nur fünf Gruppierungen im Rat, während auch bei niedriger Wahlbeteiligung eine hohe Ausdifferenzierung zu verzeichnen ist. Dies lässt den Schluss zu, dass der Wegfall der Fünf-ProzentKlausel, der für mehr Gruppierungen einen Einzug in den Rat wahrscheinlich machte, die Motivation, zur Wahl zu gehen, nicht erhöht hat, wohl aber die Motivation für potentielle Kandidaten, sich von ihren Parteien zu lösen und im Rahmen einer neuen Gruppierung zur Wahl anzutreten.

\section{Wablen der (Ober-)Bürgermeister}

Haben sich die Kommunalwahlgesetze für die Wahl der Gemeinderäte noch nicht angeglichen, so gilt dies aber deutlicher für die Wahl der (Ober-)Bürgermeister. Im Zuge der Reformen nach der Wiedervereinigung in den 1990er Jahren wurde die Einköpfigkeit der Verwaltungsspitze sowie die direkte Wahl der (Ober-)Bürgermeister nach dem Vorbild der süddeutschen Kommunalverfassung festgelegt. ${ }^{45}$ Allerdings sind die Amtsperioden für die Chefs der Verwaltung unterschiedlich (fünf bis acht Jahre), so dass die Wahlen der (Ober-) Bürgermeister in 25 Fällen bei den letzten Kommunalwahlen in Groß- und Mittelstädten nicht gleichzeitig mit denen des Kommunalparlaments stattfanden, dessen Wahlperioden fünf oder sechs Jahre betragen. ${ }^{46}$ Hinzu kommen persönliche und politische Gründe (zum

42 Vgl. Sigrid Roßteutscher / Phillip Scherer, a.a.O. (Fn. 32), S. 77.

43 Vgl. ebenda.

44 Vgl. Uwe Andersen / Rainer Bovermann, a.a.O. (Fn. 15), S. 14.

$45 \mathrm{Zu}$ Einzelheiten vgl. Hiltrud Naßmacher / Karl-Heinz Naßmacher, a.a.O. (Fn. 22), 2. Auflage, S. $201-206$.

46 In NRW wurden inzwischen die Wahlen von (Ober-)Bürgermeistern und Rat wieder zusammengelegt und als Angebot der rot-grünen Landesregierung für 2014 gilt, dass sich für sechs Jahre 
Beispiel Abwahl während der Amtszeit) für das Ausscheiden der Führungspersönlichkeiten. Die vorzeitige Abwahl kennt Baden-Württemberg ebenso wenig wie Bayern, in anderen Bundesländern wurde sie inzwischen in die Gemeindeordnungen aufgenommen. ${ }^{47}$ Der spektakulärste Fall ist die Abwahl des Duisburger Oberbürgermeisters 2012 als Folge seines Verhaltens nach dem schweren Loveparade-Unglück. In Nordrhein-Westfalen und Niedersachsen geht es immer wieder darum, ob bei der Wahl des (Ober-)Bürgermeisters beim Verfehlen der absoluten Mehrheit eines Kandidaten im ersten Wahlgang ein zweiter Wahlgang zwischen den Kandidaten mit den meisten Stimmen folgen muss (Stichwahl) oder ob der Kandidat mit den meisten Stimmen beim ersten Wahlgang die angestrebte Position erreicht hat. Nach Regierungswechseln hat die SPD immer die Stichwahl durchgesetzt und die CDU diese abgeschafft. ${ }^{48}$ Eine Variante in anderen Bundesländern ist der zweite Wahlgang, in dem alle Kandidaten noch einmal antreten und sich weitere zur Wahl stellen können (zum Beispiel in Baden-Württemberg und Sachsen). Meistens verzichten Bewerber, die im ersten Wahlgang nur wenig Wählerresonanz fanden, auf eine weitere Kandidatur. Jedenfalls genügt für den Gewinn der Position dann eine einfache Mehrheit.

Zunächst kann davon ausgegangen werden, dass das Personalangebot für diese Spitzenposition in den Städten und Gemeinden von größerer Bedeutung für die Wahlentscheidung ist als programmatische Aussagen beziehungsweise Kommunalwahlprogramme der Parteien. Allerdings lässt sich die Frage, ob Personen- und/oder Sachfragen bei Wahlentscheidungen wichtiger werden, immer noch nicht schlüssig beantworten. Allgemein scheint Sachfragen eine geringere Bedeutung zuzukommen. ${ }^{49}$

\subsection{Rolle der Parteien bei (Ober-)Bürgermeisterwahlen}

Die Direktwahl des (Ober-)Bürgermeisters sollte auch Kandidaten von außerhalb und nicht nur solche aus der örtlichen Kommunal- und damit auch Parteipolitik zur Bewerbung animieren. Da die in der Stadt vorhandene Auswahl zwangsläufig begrenzt ist, wurden so auch qualifiziertere Bewerber erwartet. Zusätzlich sollte der Parteieneinfluss geschwächt werden. 50 In Baden-Württemberg waren einmal ins Amt gewählte (Ober-)Bürgermeister Jahrzehnte lang ohnehin sicher, dass Gegenkandidaten kaum eine Chance hatten, sie aus dem Amt zu drängen, auch bei wiederholter Amtszeitverlängerung. Dies gilt seit 1973 nicht mehr uneingeschränkt. Zwar ist die Zahl der Abgewählten immer noch prozentual gering, sie nimmt jedoch zu. ${ }^{51}$ Allerdings sind davon vor allem die Amtsinhaber kleiner Städte und Gemeinden

gewählte Ober-(Bürgermeister) mit dem Rat freiwillig ein Jahr früher zur Wiederwahl stellen können. Vgl. Frank Uferkamp, Die Chance für starke Figuren, in: Solinger Tageblatt vom 21. November 2012, S. 2.

47 Vgl. Timm Kern, Warum werden Bürgermeister abgewählt?, Stuttgart 2008, S. 42.

48 Vgl. Ministerium für Inneres und Kommunales des Landes Nordrhein-Westfalen, Gesetz zur Wiedereinführung der Stichwahl vom 3. Mai 2011, http://www.mik.nrw.de/themen-aufgaben/buergerbeteiligung-wahlen (Abruf am 3. November 2012).

49 Vgl. Rüdiger Schmitt-Beck, Empirische Wahlforschung in Deutschland: Stand und Perspektiven zu Beginn des 21. Jahrhunderts, in: ders. (Hrsg.), Wählen in Deutschland, PVS-Sonderheft 45, Wiesbaden 2012, S. 2 - 39, S. 9 f.

50 Vgl. Hiltrud Naßmacher / Karl-Heinz Naßmacher, a.a.O. (Fn. 22), 2. Auflage, S. 203.

51 Vgl. Timm Kern, a.a.O. (Fn. 47), S. 9, S. 73 f. 
betroffen. 52 Bis 2003 wurden in den Städten mit über 100.000 Einwohnern Oberbürgermeister nur in Reutlingen (1993 und 2003)53 und Pforzheim (2001) nicht im Amt bestätigt.

In den Städten mit unter 100.000 Einwohnern fand in Sindelfingen 199354 sowie in Baden-Baden 1998 und Friedrichshafen 2001 jeweils eine Abwahl statt. ${ }^{55}$ Der Wahlausgang in Friedrichshafen mit sehr knappem Ergebnis zugunsten des Herausforderers (Lehrer, Journalist) wurde als Sensation gewertet und die niedrige Wahlbeteiligung dafür verantwortlich gemacht. Ein SPD-Kandidat hatte dabei einen 16 Jahre lang amtierenden Oberbürgermeister „vom Sockel gestoßen“56. In Baden-Baden konnte eine parteilose Herausforderin mit Unterstützung von FDP, Grünen und Teilen der SPD die Wahl gewinnen und einen CDUOberbürgermeister ablösen. 57

Auch die Abwahlen in Städten mit mehr als 100.000 Einwohnern fanden nicht nur landesweite Beachtung. In Reutlingen wurde ein CDU-Oberbürgermeister durch eine parteilose Kandidatin abgelöst, die allerdings von SPD, FDP, den Grünen und einer Abspaltung der CDU unterstützt wurde. 58 Der bundesweit bekannte SPD-Oberbürgermeister von Pforzheim hatte sich dadurch unglaubwürdig gemacht, dass er zweimal ohne Erfolg versuchte, eine andere Spitzenposition zu erlangen. Am aufsehenerregendsten war seine Kandidatur für das Amt des Oberbürgermeisters von Stuttgart. Dieser Alleingang kostete ihn auch den Rückhalt in seiner eigenen Partei. Es gewann in Pforzheim 2001 die FDP-Kandidatin, eine langjährige Stadträtin, die von der CDU unterstützt wurde. 59

Dagegen wurde in Nordrhein-Westfalen 1999 die erste Chance, den Oberbürgermeister direkt zu wählen, von den Wählern genutzt, um acht Amtsträger in 23 kreisfreien Städten, die nach der Reform 1994 - zunächst durch die Räte - gewählt waren ${ }^{60}$, wieder aus dem Amt zu jagen. Zwei Beispiele aus der Oberbürgermeisterwahl im Jahre 1999 wurden intensiver untersucht: Die durch Ratswahl ins Amt gekommene Oberbürgermeisterin in der SPD-dominierten Stadt Duisburg wurde noch durch die Direktwahl bestätigt. In der Nachbarstadt Essen - ebenfalls eine Hochburg der SPD - gelang dies nicht. Vermutet werden weiterschwelende parteiinterne Konflikte in der SPD, die zur mangelhaften Unterstützung des nach kontroversen Diskussionen in die Wahl geschickten Kandidaten führten. ${ }^{61}$ Werden Städte aller Größenordnungen einbezogen, so besetzte die CDU nach der Wahl 1999 zwei Drittel aller kommunalen Spitzenämter. ${ }^{62}$

Den Einfluss der Parteien auf der kommunalen Ebene zurückzudrängen scheint durch die Direktwahl in Nordrhein-Westfalen zumindest in den Städten mit über 50.000 Einwoh-

52 Vgl. ebenda, S. $111 \mathrm{ff}$.

53 Vgl. ebenda, S. 88, S. 94.

54 Vgl. ebenda, S. 88, S. 92.

55 Vgl. ebenda, S. 93, S. 145, S. 164.

56 Ebenda, S. 145, aufgrund der Berichterstattung des Südkuriers.

57 Vgl. ebenda, S. 120 f.

58 Vgl. ebenda.

59 Vgl. ebenda, S. $235 \mathrm{f}$.

60 Dies ergab sich dadurch, dass die Verwaltungschefs in den Ruhestand gingen, sich beruflich veränderten oder ihre Amtszeit auslief. Keiner derjenigen, die durch den Rat gewählt waren, hat auf die Kandidatur bei der Direktwahl verzichtet. Hiltrud Naßmacher, a.a.O. (Fn. 12), S. 24 ff.

61 Vgl. Uwe Andersen / Rainer Bovermann, a.a.O. (Fn. 15), S. 32; Lars Holtkamp / David H. Gehne, Bürgermeisterkandidaten zwischen Verwaltungsprofis, Parteisoldaten und Schützenkönigen, in: Uwe Andersen / Rainer Bovermann, a.a.O. (Fn. 11), S. 55 - 88, S. 58, S. 63 f.

62 Vgl. Uwe Andersen / Rainer Bovermann, a.a.O. (Fn. 15), S. 21. 
nern kaum gelungen zu sein. Das Beispiel Baden-Württembergs, wo Parteien immer schon in kleinen Gemeinden keine große Rolle spielten, ist bei Betrachtung mittlerer und größerer Städte kaum übertragbar. Obwohl die Parteien in Baden-Württemberg keine Rechte bei der Kandidatenaufstellung haben und auf die parteipolitische Unabhängigkeit der Bewerber Wert gelegt wird63, sind Kandidaten bereits in Städten mittlerer Größe im Wahlkampf auf die Unterstützung der Parteien zur Mobilisierung der Wähler angewiesen. Der Einfluss der Parteien bei den Bürgermeisterwahlen wird „mit wachsender Einwohnerzahl immer größer"64. Dies sichert Parteien und politischen Gruppierungen auch hier eine entscheidende Mitsprache bei der Auswahl der Kandidaten. In Nordrhein-Westfalen ist der Einfluss der Parteien auf die Auswahl der Kandidaten ungebrochen. Sie beherrschen weitgehend den Zugang zum Oberbürgermeisteramt. Dort, wo CDU und FDP 1999 aus taktischen Gründen (wegen geringer Wählerresonanz in den vorangegangenen Kommunalwahlen) einen unabhängigen Bewerber unterstützten, hatte dieser keine Chance, zum Beispiel in Duisburg 1999.65

Zwar nimmt die Prägekraft der „cleavages“ bei den Wahlen ab, allerdings zeigen sie offenbar doch noch Wirkung bei der Mobilisierung und als Entscheidungshilfe. ${ }^{66}$ Dies wird natürlich besonders deutlich, wenn die Wahlen zum Kommunalparlament und die Oberbürgermeisterwahlen zeitlich zusammenfallen. ${ }^{67}$ Bei Betrachtung der aktuellen Wahlergebnisse aller Bundesländer in Städten über 50.000 Einwohner zeigt sich, dass in den seltensten Fällen dort, wo CDU oder CSU die Mehrheit im Rat haben (über 40 Prozent Wählerresonanz bei den letzten Wahlen zu den Kommunalparlamenten), der (Ober-)Bürgermeister einer anderen Partei angehört. Nur in Rheine und Viersen ist er Mitglied der SPD, in Bad Homburg v.d.H. der Grünen, in Neu-Ulm der CSU. Die CDU-Hochburg Lingen hat einen parteilosen Oberbürgermeister. 68

Dies gilt entsprechend, wenn die SPD die meisten Stimmen bei der Wahl zum Kommunalparlament auf sich vereinigen konnte. Dann gehört der Oberbürgermeister dieser Partei an. Eine Ausnahme bildet beim Zusammenfallen der Wahl zum Kommunalparlament und des Oberbürgermeisters nur Ludwigshafen. Hier konnte eine CDU-Kandidatin mit einer Vorbildung, die dem typischen Anforderungsprofil für dieses Amt in Baden-Württemberg entspricht (Juristin mit Verwaltungserfahrung), 2001 erstmals die sozialdemokratischen Stadtoberhäupter ablösen. Sie wurde 2009 wiedergewählt. ${ }^{69}$

In den anderen Städten mit der SPD als stärkster Partei im Rat und einem (Ober-)Bürgermeister einer anderen Partei fielen die beiden Wahlen nicht zusammen, so dass CDUOberbürgermeister in Salzgitter und Wolfenbüttel, ein Oberbürgermeister der FDP in Wetzlar und parteilose (Ober-)Bürgermeister in Cuxhaven, Hameln und Oldenburg amtieren. Allerdings kam die SPD bei den letzten Wahlen zu den Kommunalparlamenten (2008,

63 Vgl. Timm Kern, a.a.O. (Fn. 47), S. 45, S. 55.

64 Ebenda, S. 118.

65 Vgl. Lars Holtkamp / David H. Gehne, a.a.O. (Fn. 61), S. 64 f.

66 Vgl. Anja Neundorf, Die Links-Rechts-Dimension auf dem Prüfstand: Ideologische Einstellungen und Wahlverhalten im vereinten Deutschland 1990 bis 2008, in: Rüdiger Schmitt-Beck, a.a.O. (Fn. 49), S. 233 - 256, S. 246.

67 Vgl. Rainer Bovermann, a.a.O. (Fn. 15), S. 115 - 159, S. 137.

68 Eigene Auszählungen nach Konrad Adenauer Stiftung (Hrsg.), a.a.O. (Fn. 18).

$69 \mathrm{Vgl}$. Homepage der Stadt Ludwigshafen, http://www.ludwigshafen.de/buergernah/rathaus/oberbuergermeisterin-dr-eva-lohse/ (Abruf am 21. Januar 2013). 
2009, 2012) selten auf über 40 Prozent der Stimmen, nämlich nur in insgesamt 18 Städten (50.000 bis über 100.000 Einwohner). Davon befinden sich zwölf in Nordrhein-Westfalen, zwei in Niedersachsen und drei in Bayern. ${ }^{70}$ In 35 Städten konnte die SPD auch dann den (Ober-)Bürgermeister stellen, wenn sie zweit- (in 30 Städten), drittstärkste (Chemnitz, Leipzig, Villingen-Schwenningen und Zwickau) und sogar nur fünftstärkste Partei (Weimar) im Kommunalparlament war. ${ }^{71}$ Dies weist auf Bündnisse von Parteien für einen Kandidaten hin oder darauf, dass sich die Mehrheitsverhältnisse im Rat durch zwischenzeitliche Neuwahlen geändert hatten. Kaum ist anzunehmen, dass ein Kandidat so herausragend war, dass er im Wahlkampf als Einzelkämpfer Erfolg haben konnte. Hier müssten schon Amtsbonus und besondere Qualifikation zusammenkommen.

Bei den Grünen haben die Oberbürgermeister von Darmstadt, Freiburg i.Br., Stuttgart und Tübingen jeweils die stärkste Fraktion im Rat hinter sich, nicht aber in Bad Homburg.

Bei quantitativer Analyse ergibt sich, dass im Oktober 2012 in Städten über 100.000 Einwohner fast doppelt so viele SPD-Oberbürgermeister an der Spitze der kommunalen Verwaltung standen (nämlich 41) wie solche mit CDU-Mitgliedschaft (24). Parteilose (acht) und solche, die sich zu den Grünen bekennen (drei) hatten vor allem in Universitätsstädten Erfolg. Hinzu kommen Erfolge solcher Kandidaten in Baden-Württemberg und den neuen Bundesländern. ${ }^{72}$

In den Städten mit unter 100.000 bis 50.000 Einwohner ist die Anzahl der (Ober-) Bürgermeister für die großen Parteien fast gleich: Die SPD stellt 46, die CDU/CSU 40 Führungskräfte der Kommunen. Allerdings gewinnt die CSU diese vor allem in kleineren Städten. Insgesamt kamen in den kleineren Städten auch parteilose Kandidaten (15) und solche der Grünen (zwei) sowie von Wählergemeinschaften oder landesspezifischen Parteien (FW, SSW) ins Amt. Parteilose waren wie bei den größten Städten in den neuen Bundesländern sowie in Baden-Württemberg und Bayern erfolgreich. Die Linken stellen in zwei Städten Ostdeutschlands den Oberbürgermeister (Eisenach und Schwerin). ${ }^{73}$

\subsection{Stadtspezifische Einflüsse bei (Ober-)Bürgermeisterwahlen}

Die bisherigen Ergebnisse zeigen eindeutig, dass die Parteien und ihr Engagement im Wahlkampf wichtig sind. Wenn Parteien auf der lokalen Ebene nach Kommunalwahlen die stärksten Fraktionen in den Rat schicken konnten, gelang es ihnen in der Regel auch, ihrem (Ober-)Bürgermeisterkandidaten zum Sieg zu verhelfen. Die oben angeführten Ausnahmen von der Regel lassen auf stadtspezifische Ursachen schließen.

Die SPD hatte bei den Kommunalwahlen in Nordrhein-Westfalen im Jahr 1999 erdrutschartige Stimmenverluste. Die Ursache wurde schnell im bundesweiten Tief der Partei während des ersten Jahres der rot-grünen Koalition gesehen. ${ }^{74} \mathrm{Im}$ Vergleich dazu war die Lage der CDU im Bund kurz vor der Bundestagswahl 2013 - trotz häufiger Kritik am Zustand der Koalition mit der FDP - stabil. Dies liegt auch an der Beliebtheit der Kanzlerin.

70 Vgl. Konrad Adenauer Stiftung (Hrsg.), a.a.O. (Fn. 18), S. 8 - 52.

71 Vgl. ebenda, S. 50, S. 52 f.

72 Eigene Auszählungen nach ebenda, S. 8 - 26.

73 Vgl. ebenda, S. $27-52$.

74 Vgl. Uwe Andersen / Rainer Bovermann, a.a.O. (Fn. 15), S. 7, S. 18. 
Hier kann also die Ursache für die Wahlniederlagen der CDU im Jahr 2012 in den großen Städten Frankfurt, Stuttgart, Karlsruhe und Kiel sowie 2013 in Leipzig nicht gesucht werden. In Nordrhein-Westfalen gab es 1999 nach den Wahlen nur vereinzelt Hinweise darauf, dass die jahrelange Dominanz der SPD und die von Wahlberechtigten wahrgenommenen Probleme in einzelnen Städten möglicherweise am Wahldesaster mit schuldig waren. Seltener wurde von offizieller Seite an der Qualität der Kandidaten für die Spitzenpositionen gezweifelt. ${ }^{75}$

Die Kandidaten bei der Wahl 1999 in Nordrhein-Westfalen kamen alle aus den engeren kommunalpolitischen (bis dahin „ehrenamtlichen“) Führungskreisen. Sie waren damit Teil des wahrgenommenen Parteienfilzes, der für Unmut der Wähler mitverantwortlich war: die Dominanz der SPD in den Städten des Ruhrgebiets. Hinzu kamen einzelne stadtspezifische Skandale in Dortmund und Köln. Der Verdruss darüber mag sich beim Abstimmungsverhalten Bahn gebrochen haben: Die SPD-Kandidaten wurden aus Protest nicht gewählt. Bewerber von außerhalb kamen nicht zum Zuge. Manche mutmaßten, dass diese Konkurrenten auch gar nicht gesucht wurden oder dass die kurze Wahlzeit von fünf Jahren für die Oberbürgermeister in Nordrhein-Westfalen kein Anreiz für auswärtige Bewerber zur Kandidatur gewesen sei. Es ist nicht auszuschließen, dass die mehrheitlich langfristig aufgebauten Startnachteile durch besser geeignete Kandidaten zumindest teilweise hätten wettgemacht werden können.

\subsection{Bedeutung der (Ober-)Bürgermeisterkandidaten}

Die genannten Ausnahmen von der Regel, dass die Fraktion mit den meisten Ratsmitgliedern auch den Oberbürgermeister stellt, wiesen bereits auf die Bedeutung der Kandidaten für die Wahlen hin. Daneben kann davon ausgegangen werden, dass Unzufriedenheit mit der Politik einer dominanten Partei vor Ort eine wichtigere Rolle spielt. Für die Wiederwahl eines Oberbürgermeisters ist die Performanz im Amt ohnehin von entscheidender Bedeutung, weil er der am meisten wahrgenommene Repräsentant der Stadt (und zuweilen auch der führenden Partei) ist.

\section{(1) Berufsspezifische Qualifikation der Kandidaten}

Ein Indiz für die Bedeutung dieser Qualifikation ist, dass einzelne Kandidaten 1999 und 2004 in Nordrhein-Westfalen gegenüber ihrer Partei erhebliche Stimmenvorsprünge im zweistelligen Bereich erzielen konnten ${ }^{76}$, denn die meisten Oberbürgermeisterkandidaten erhielten kaum mehr Stimmen als ihre Partei. Dagegen ragte besonders das Ergebnis des Kandidaten in Aachen heraus, der 1999 20,2 Prozent und 200429 Prozent mehr Stimmen als seine Partei erhielt. Er hatte neben der Ortsverbundenheit und politischen Erfahrung auch eine berufliche Qualifikation als Rechtsanwalt und Notar vorzuweisen, die ihn als sachkompetent für die Führungsfunktion auswies. Weitere ebenfalls beruflich für Führungspositionen Qualifizierte gingen in Bonn und Wuppertal mit großem Vorsprung an Wähler- 
stimmen vor ihrer Partei aus der Abstimmung hervor. 77 Auch in Bonn konnte die Oberbürgermeisterin 2004 noch mit 27,2 Prozent (gegenüber 17,6 Prozent 1999) beim Vorsprung zulegen. Dagegen muss bei manchen durch den Rat gewählten und 1999 abgewählten Oberbürgermeistern die Frage der Qualifikation für das Amt gestellt werden. Ist politische Managementerfahrung und Ortskenntnis bereits ausreichend, oder sollte sie durch ein juristisches oder verwaltungswissenschaftliches Studium zusätzlich fundiert sein? Zumindest für Baden-Württemberg, vor allem im Landesteil Württemberg, wurde die Fachkompetenz als bedeutend herausgearbeitet. ${ }^{78}$

Von diesen Qualifikationsprofilen wichen die Bewerber in Nordrhein-Westfalen zumindest bei den ersten Direktwahlen deutlich ab. ${ }^{79}$ Vielen Bewerbern half der Amtsbonus ${ }^{80}$ zumindest im ersten Wahlgang. Fehlverhalten der Oberbürgermeister wird in der Regel vom Wähler geahndet, so 1999 zum Beispiel in Dortmund und Köln.81 Dies war auch bei der Oberbürgermeisterwahl 2004 in Wuppertal der Fall. ${ }^{22}$ Gegen den fachlich sehr gut qualifizierten, zur Wiederwahl stehenden Amtsinhaber, der noch 1999 mit deutlichem Vorsprung vor seiner Partei im ersten Wahlgang gewonnen hatte, gab es Korruptionsvorwürfe wegen Parteispenden durch einen Bauunternehmer. Insgesamt zeigten die Wahlen 2004, dass sich eine Verliererin bei vorangegangenen Wahlen auch wieder in der Wählergunst rehabilitieren kann. So konnte die SPD im Ruhrgebiet wieder mehr Spitzenpositionen erlangen als die CDU: „Insbesondere bei den Stichwahlen schlug das Pendel zugunsten der SPD aus. Dabei spielten eindeutig stadtspezifische Ursachen mit hinein." 83

Der Einfluss des Personalangebots auf den Ausgang der Wahl sollte also nicht unterschätzt werden. Dabei geht es allerdings nicht nur um die fachliche Qualifikation. Kern stellte etwa für Baden-Württemberg fest, dass Verwaltungserfahrung innerhalb von 30 Jahren an Bedeutung verloren hat und dass stattdessen kommunikative Fähigkeiten heute von größerer Bedeutung sind. ${ }^{84}$ In Essen gewann 2009 - dem Milieu der Ruhrgebietsstadt eher angemessen - wieder ein Kandidat der SPD, der wenig in das von den Reformern der Wahl angepeilte Anforderungsprofil bezüglich seiner Berufsausbildung passte. Er ist Ingenieur (Fachrichtung Chemie) und war bisher Fraktionsvorsitzender im Rat. ${ }^{85}$ Hier zeigt sich in Nordrhein-Westfalen, dass nach wie vor Kandidaten ohne Verwaltungs-, aber mit politischer Managementerfahrung (Fraktionsvorsitzende der stärksten Fraktion) Chancen auf das Amt des Oberbürgermeisters haben. Ein weiteres Beispiel dafür ist der 2009 gewählte Oberbürgermeister von Aachen (2009), zunächst Handwerksmeister, dann Unternehmer. ${ }^{86}$ Auch Beispiele solcher Personen, denen sichere Wiederwahlen gelangen, sind zu nennen, etwa der Ober-

77 Vgl. ebenda, S. $25 \mathrm{ff}$.

78 Vgl. Hans-Georg Wehling / H. Jörg Siewert, a.a.O. (Fn. 9).

79 Vgl. Lars Holtkamp / David H. Gehne, a.a.O. (Fn. 61).

80 Dies kann auch bei der Wahl 2004 festgestellt werden. Vgl. Hiltrud Naßmacher, a.a.O. (Fn. 12), S. 30 .

81 Vgl. ebenda, S. 27.

82 Vgl. ebenda, S. 30.

83 Ebenda, S. $29 \mathrm{f}$.

84 Vgl. Timm Kern, a.a.O. (Fn. 47), S. 356.

85 Vgl. Lebenslauf von Essens Oberbürgermeister Reinhard Paß, http://www.essen.de/de/Rathaus/ Oberbuergermeister/Vita.html (Abruf am 20. Januar 2013).

86 Vgl. Lebenslauf von Aachens Oberbürgermeister Marcel Philipp, http://www.aachen.de/DE/ stadt_buerger/oberbuergermeister/lebenslauf/index.html (Abruf am 20. Januar 2013). 
bürgermeister von Hamm, ehemals Metzgermeister ${ }^{87}$ und von Recklinghausen, ehemals Berufsschullehrer. ${ }^{88}$ Die Listen der Mitgliedschaften dieser Persönlichkeiten zeigen eine starke Vernetzung in der Stadt.

\section{(2) Gegenkandidaten}

Natürlich spielen auch die Gegenkandidaten eine Rolle. So gewann die CDU bei der gleichzeitigen Ratswahl in Iserlohn zwar die meisten Stimmen. Der direkt gewählte Oberbürgermeisterkandidat der SPD konnte aber mehr Stimmen auf sich vereinigen als seine Partei. Seine langjährige Verwaltungstätigkeit als Stellvertreter des Bürgermeisters und Leiter des Ressorts „Planen, Bauen und Wohnen“ sowie seine Lehrtätigkeit im Fach Volkswirtschaftslehre wiesen ihn zudem als sachkompetent aus. Der CDU-Konkurrent für das Amt - offenbar ein Autohauseigentümer - hatte schon bei der Nominierung durch die CDU Schwierigkeiten, so dass davon auszugehen ist, dass die Mobilisierung der Partei für diesen Kandidaten eher mäßig war. ${ }^{89}$

In personenbezogenen Wahlkämpfen kommt es auch auf die soziale Kompetenz des Kandidaten oder Amtsinhabers an. ${ }^{90}$ So waren beim Essener CDU-Oberbürgermeister die fachlichen Voraussetzungen durch seinen Beruf als Rechtsanwalt und Notar sowie langjährige politische Erfahrungen zweifellos vorhanden. Er war 1999 sensationell gegen den hoch favorisierten SPD-Kandidaten gewählt und auch $2004 \mathrm{im}$ Amt bestätigt worden, verzichtete aber 2009 auf eine erneute Kandidatur. Trotz partei- und kommunalpolitischer Erfahrung als langjähriges Ratsmitglied blieb er aber in seiner Oberbürgermeisterzeit der kompetente Anwalt. In einem Kommentar wird er als „nüchterner Jurist“, „,entscheidungsschwach und unpolitisch" bezeichnet, der einen eleganten Abgang gefunden habe. ${ }^{91}$ In Oldenburg92 konnte 1996 ein Jurist mit Verwaltungserfahrung in höherrangiger Position zwar gegen einen bisherigen ehrenamtlichen Oberbürgermeister gewinnen, der vor allem Erfahrung in der örtlichen Kommunalpolitik vorweisen konnte, aber nicht in Verwaltungsaufgaben. Die Wiederwahl des Verwaltungsjuristen scheiterte 2001 allerdings nach einer Wahlperiode wegen fehlender sozialer Kompetenz. Ihm wurde angekreidet, dass er als Beamter auf geregelte Arbeitszeit Wert lege und damit zu wenig soziale Kontakte pflege sowie seinen Wohnsitz in der Nachbargemeinde beibehielt. Der Gegenkandidat war auch Jurist, zudem politisch versiert, in Oldenburg geboren und dort wohnhaft. Die Persönlichkeiten der Kandidaten sind sicher wichtig. Aber nur auf die Person zu setzen, unter anderem ihre „unpolitischen Per-

87 Vgl. Lebenslauf von Hamms Oberbürgermeister Thomas Hunsteger-Petermann, http://www.hamm. de/rathaus/ob/obpersoenlich/biographie.html (Abruf am 2. Dezember 2012).

88 Vgl. Lebenslauf von Recklinghausens Bürgermeister Wolfgang Pantförder, http://www.recklinghausen.de/Inhalte/Startseite/Rathaus_Politik/Buergermeister/index.asp?highmain=3\&highsub=0\&h ighsubsub=0 (Abruf am 2. Dezember 2012).

89 Vgl. SPD-Wahlportal der Städte und Gemeinden des Märkischen Kreises, http://www.spdmkwahlportal.de (Abruf am 3. Dezember 2012); http://www.derwesten.de/ikz/staedte/iserlohn/ hartmut-bogatzki-cdu-bue... (Abruf am 3. Dezember 2012).

90 Vgl. Timm Kern, a.a.O. (Fn. 47), S. 231.

91 Tobias Blasius, Abschied von Essens OB Reiniger nach zehn Jahren, in: WAZ.de, http://www. derwesten.de/staedte/essen/abschied-von-essens-ob-reiniger-nach-zehn-jahren-id20281.html (Abruf am 9. Januar 2013).

92 Eigene Beobachtungen bei jahrelanger politischer Aktivität in der Stadt. 
sönlichkeitsmerkmale“, scheint nur in Einzelfällen aussichtsreich, denn es geht nicht zuletzt um ihre Rolle bei politischen Entscheidungen auf der kommunalen Ebene.

\subsection{Bedeutung der Sachfragen bei (Ober-)Bürgermeisterwahlen}

Einzelne politische Entscheidungen und das überörtliche Engagement können sehr große Unzufriedenheit hervorrufen, die von konkurrierenden Parteien, von Vereinen, Verbänden und neuen Initiativen öffentlichkeitswirksam aufgegriffen wird. Dies war zum Beispiel in Oldenburg der Fall. Der SPD-Oberbürgermeister hatte den Bau eines innerstädtischen Einkaufszentrums vorangetrieben und die baurechtlichen Weichen gestellt. Eine Bürgergruppierung und die Grünen lehnten den Bau als problematisch für die gewachsene Innenstadtstruktur ab. Der von außerhalb durch die CDU rekrutierte Kandidat, parteilos und zudem durch seine Titel (Prof. Dr.) wohl als besonders kompetent eingeschätzt, schlug sich auf die Seite der Kritiker des Einkaufszentrums. Er versprach, die Umsetzung zu verhindern und wurde fortan von den Grünen unterstützt. Der Amtsinhaber verfehlte 2006 die absolute Mehrheit im ersten Wahlgang äußerst knapp. In der Stichwahl gewann der von CDU und Grünen unterstützte Kandidat. Der neu gewählte Oberbürgermeister konnte den Bau des Einkaufszentrums nicht verhindern, weil der Entscheidungsprozess schon viel zu weit fortgeschritten war. Nur eine Reduzierung des Bauvolumens war noch möglich. Alle Vermutungen laufen darauf hinaus, dass die Wähler (und insbesondere die Parteiaktivisten) bei einer erneuten Kandidatur diesen „Wahlbetrug“ ahnden werden. Die Unzufriedenheit mit dem Amtsinhaber erstreckt sich auch auf weitere Aktivitäten und wird nicht nur von den Fraktionen im Rat (mit Ausnahme der CDU), sondern auch von Bürgern bei Befragungen immer wieder artikuliert. Zweimal (2009 und 2011) waren von den Grünen initiierte Abwahlverfahren allerdings an der fehlenden Dreiviertelmehrheit im Rat als erster Stufe gescheitert.

Für Baden-Württemberg wird konstatiert, dass sich viele Bürgermeister auch deshalb in eine Partei einbinden lassen, weil sie auf der Kreisebene aktiv sein wollen ${ }^{93}$, um als Mitglied des Kreistages Informationen zu generieren, die für die Entwicklung der Gemeinde von Bedeutung sein könnten. Bei größeren Städten tritt an diese Stelle die Kooperation mit Umlandgemeinden in mehr oder weniger formalisierten Gremien. Hier schließen sich größere Städte mit gleichen Problemen (zum Beispiel die Ruhrgebietsstädte oder die des Bergischen Landes) oder Stadt-Umland Gemeinden zu einer zwar formalisierten, allerdings in der Regel auf Einzelaufgaben bezogenen Kooperation zusammen - allerdings unter Wahrung der eigenen Interessen. Es geht immer mehr um einen Informationsaustausch. Die (Ober-) Bürgermeister müssen dabei eine Balance finden zwischen dem Zeiteinsatz für Aktivitäten auf der örtlichen Ebene und der Pflege von überörtlichen Kontakten mit Kooperationsoptionen und der Möglichkeit, stadtentwicklungsrelevante Informationen zu erhalten.

Der Oldenburger Oberbürgermeister zum Beispiel pflegt die unmittelbaren Umlandkontakte eher dilatorisch. Dagegen hat er ein Faible für die Zusammenarbeit mit China. Seine China-Exkursionen mit Begleitern und eine entsprechende Anlaufstelle innerhalb der Verwaltung bringen ihm regelmäßig kritische Kommentare aus dem Rat ein, die auch in der Lokalpresse unterstützt werden. Bei Bürgerbefragungen wird die Kooperation ebenfalls sehr

93 Vgl. Timm Kern, a.a.O. (Fn. 47), S. 2. 
kritisch angemerkt. ${ }^{94}$ Inzwischen hat der Oberbürgermeister auf eine erneute Kandidatur für das Amt verzichtet. 95 Seine Wiederwahlchancen wären gewiss auch beeinflusst durch die absehbaren Gegenkandidaten und die Wahlbeteiligung im zweiten Wahlgang.

\subsection{Wahlbeteiligung im zweiten Wahlgang}

Die Nichtbeteiligung an den Direktwahlen der Oberbürgermeister ist in größeren Städten besonders hoch. Für Baden-Württemberg stellte Timm Kern fest $^{96}$, dass die Wahlbeteiligung mit steigender Einwohnerzahl sinkt. In Niedersachsen ging sie bereits kurz nach Einführung der Direktwahlen erheblich zurück. Der zweite Wahlgang scheint für die Wiederwahl von Amtsinhabern eine besondere Gefahr darzustellen. Ob das Risiko bei der Stichwahl zwischen den Bestplatzierten des ersten Wahlgangs oder als quasi Wahlwiederholung (mit der Möglichkeit für weitere Bewerber, zusätzlich anzutreten) höher ist, lässt sich kaum generell abschätzen. Es ist aber eine Tatsache, dass Kandidaten, die im ersten Wahlgang aufgrund ihrer Wählerresonanz als wenig aussichtsreich gelten, ihre Kandidatur zurückziehen und dann an ihre Wähler appellieren, die Stimme einem aussichtsreicheren Bewerber zu geben. Neue Kandidaten im zweiten Wahlgang hatten bisher keine Chance.

Auch profilierte Oberbürgermeister sind bei Stichwahlen gescheitert, vermutlich weil der Amtsinhaber selbst von einem sicheren Sieg ausgegangen war, die Chancen des Herausforderers unterschätzte und daher eine besondere Mobilisierung seiner Wähler für den zweiten Wahlgang unterließ. Dies hat (neben der bereits vorgestellten kontroversen Sachfrage) nach Einschätzung der Beobachter aus der engeren Wahlkampfführung97 die Wiederwahl des Oldenburger Oberbürgermeisters gekostet, der trotz der dargestellten Streitfrage (Einkaufszentrum) als hoher Favorit in die Wahl gegangen war.

Der Oldenburger Fall von 2006 war aber keine Besonderheit, denn auch in Salzgitter wurde im selben Jahr ein SPD-Oberbürgermeister (ehemals Lehrer) durch einen Verwaltungsfachmann der CDU abgelöst, vermutlich wegen unzureichender Mobilisierung der Anhänger für die Stichwahl. 98 Beide Herausforderer wurden für acht Jahre gewählt. Der aktuellste Fall ist die Niederlage des CDU-Oberbürgermeisters von Wiesbaden in der Stichwahl im März 2013, wobei er einen Vorsprung vor seinem Hauptkonkurrenten von etwa zehn Prozentpunkten im zweiten Wahlgang nicht halten konnte. ${ }^{99}$ Auch Kern kann von vielen zum Teil spektakulären Beispielen ${ }^{100}$ berichten, allerdings für kleinere Gemeinden. Die Wähler dürfen also nicht in der Vorstellung gelassen werden, dass der Wahlausgang zugunsten des Amtsinhabers sicher ist und der zweite Wahlgang nicht so wichtig sei.

94 So führte die China-Initiative die Streichliste beim Bürgerhaushalt 2011 an. Vgl. NordwestZeitung vom 17. August 2011.

95 Der Oberbürgermeister plant seine Wahlzeit, die ihn für 8 Jahre ins Amt gebracht hat, nicht zu verlängern. Vgl. Michael Exner, Egal was kommt: Schwandner geht 2014, in: Nordwest-Zeitung vom 6. Februar 2013.

96 Vgl. Timm Kern, a.a.O. (Fn. 47), S. 153.

97 Interview mit Mitgliedern der Wahlkampfführung in Oldenburg kurz nach der Wahlniederlage.

98 http://www.salzgitter.de/rathaus/presse_news/2006/12006101000000166... (Abruf am 2. Dezember 2012).

99 Sie unten.

100 Vgl. Timm Kern, a.a.O. (Fn. 47), S. 25, S. 128 f. 
Jedenfalls sinkt die Wahlbeteiligung im zweiten Wahlgang erheblich. Eine besonders geringe Wahlbeteiligung gab es 2011 in Offenbach mit nur 24,3 Prozent der Stimmen und in Duisburg 2012 mit nur 25,8 Prozent. ${ }^{101}$ Vermutlich deswegen hatte die CDU in NordrheinWestfalen für 2009 und in Niedersachsen den zweiten Wahlgang abgeschafft. Die rot-grünen Landesregierungen in beiden Ländern (seit 2010 beziehungsweise 2013 im Amt) führten ihn wieder ein. Die geringe Wahlbeteiligung löst dann wiederum in der Lokalpresse die Debatte darüber aus, ob der mit weniger als der Hälfte der Stimmen Gewählte überhaupt ausreichend für das Amt legitimiert sei. In der Tat sind in Nordrhein-Westfalen 2009 in Städten über 100.000 Einwohner 16 Oberbürgermeister mit weit weniger als 50 Prozent der Stimmen ins Amt gekommen: etwa in Krefeld und Leverkusen mit nur 39,9 Prozent oder in Solingen mit nur 38,1 Prozent. In Niedersachsen reichten 2011 in Städten unter 100.000 Einwohnern 36,1 Prozent (Wilhelmshaven) beziehungsweise 37,6 Prozent der Stimmen (Cuxhaven) zur Wahl in eine achtjährige Amtszeit.

\section{Aktuelle Oberbürgermeisterwahlen im Lichte der bisherigen Ergebnisse}

Die in der Öffentlichkeit stark beachteten Oberbürgermeisterwahlen waren die von Karlsruhe, Frankfurt, Stuttgart, Kiel, Leipzig und Wiesbaden. In den vier erstgenannten Städten traten die bisherigen Amtsinhaber nicht mehr zur Wahl an. In Leipzig und Wiesbaden versuchte der bisherige Oberbürgermeister für eine weitere Amtszeit gewählt zu werden. Gemeinsam ist allen Wahlen, dass es keinen Sieg für die CDU-Kandidaten gab.

Die bisherigen Analysen haben gezeigt, dass die Mobilisierungsaktivitäten der Parteien sowohl für den ersten als auch für den zweiten Wahlgang sehr wichtig sind. Nimmt man die Zusammensetzung der Stadträte als Indikator, so konnte sich in den obigen Städten keiner der Kandidaten nur auf die Unterstützung der Partei verlassen, von der er aufgestellt war. In den Räten befinden sich seit den letzten Wahlen sieben (Karlsruhe, Kiel, Leipzig), acht (Stuttgart), neun (Wiesbaden) und in Frankfurt sogar elf Gruppierungen. Nur in Stuttgart (Grüne) und Kiel (SPD) konnten die Parteien mit hauchdünner (relativer) Mehrheit im Rat ihren Kandidaten durchsetzen. Die Unterstützung durch andere Parteien und Gruppierungen war also notwendig. Soll das gelingen, kommt es sehr stark auf den präsentierten Kandidaten an.

Wie bereits dargelegt müssen kommunale Führungspersönlichkeiten Fachkompetenz, politische Managementfähigkeiten und soziale Kompetenz besitzen. Sie sollten sich jedenfalls zumindest vom aussichtsreichsten Gegenkandidaten positiv unterscheiden. Eine innerparteiliche Auseinandersetzung bei der Kandidatenaufstellung kann die Bereitschaft der unterlegenen Gruppierung in den Parteien schmälern, sich im Wahlkampf zu engagieren. Eine kontrovers geführte Diskussion über eine in der kommunalen Öffentlichkeit als bedeutend propagierte Frage kann wahlentscheidend sein. Insofern ist es für die in der Diskussion

101 Vgl. Konrad Adenauer Stiftung (Hrsg.), a.a.O. (Fn. 18), S. 11, S. 20. In Duisburg waren die Wahlberechtigten sicher wahlmüde, weil sie im Abwahlverfahren gegen den 2004 und 2009 gewählten Oberbürgermeister schon gefordert waren oder „der Wunsch nach einem überparteilichen Kandidaten“ sich nicht erfüllt hatte. Schon im ersten Wahlgang mit nur 32,8 Prozent Wahlbeteiligung hatte sich dies gezeigt. Der Gewählte war bisher SPD-Landtagsabgeordneter. Vgl. Reiner Burger, Die Last des Amtes, in: FAZ vom 30. Juni 2012, S. 5. 
involvierten Kandidaten und ihre Partei besonders wichtig, Überzeugungsarbeit für den eigenen Standpunkt zu leisten, um Wähler von der Position des Kandidaten zu überzeugen.

Die Frage ist, ob diese Aspekte bei den hier betrachteten Oberbürgermeisterwahlen von den Kandidaten, ihren wahlkämpfenden Parteien und den sonstigen Anhängern der Kandidaten beachtet wurden. Bei der Wiederwahl ergibt sich nur dann ein Amtsbonus, wenn es dem Oberbürgermeister gelungen ist, seine Handlungskompetenz mit der notwendigen Überzeugungsarbeit zu begleiten und damit Vertrauen zu gewinnen.

In Karlsruhe trat der CDU-Oberbürgermeister aus Altersgründen nicht mehr an, so dass die Partei einen neuen Kandidaten präsentieren musste. Die Wahl hat allerdings der SPDKandidat gewonnen, obwohl die Sozialdemokraten nur drittstärkste Partei im Rat sind. Bei der Aufstellung des CDU-Kandidaten gab es innerparteiliche Konkurrenz. Ein Bundestagsabgeordneter und Volljurist hatte sich gegen die mit Finanzen betraute Bürgermeisterin, die als erste ihre Kandidatur angekündigt hatte, durchgesetzt. Er wurde wahrscheinlich aber von der CDU im Wahlkampf nur teilweise unterstützt. Ihm haftete „der Ruf an, intrigant und sehr ehrgeizig zu sein“102. Damit konnte nach 42 Jahren in der CDU-Hochburg Karlsruhe der SPD-Kandidat das Spitzenamt in der drittgrößten Stadt Baden-Württembergs erlangen.

Er konnte auch auf die Unterstützung der Grünen, der Piraten und der Karlsruher Bürger-Liste (KAL) zählen und gewann mit absoluter Mehrheit im ersten Wahlgang bei einer Wahlbeteiligung von 42,2 Prozent, die höher war als bei der letzten Oberbürgermeister-Wahl im Jahre 2006. Als Qualifikationen konnte er auf Erfahrungen in der Kommunalpolitik (Ratsmitglied in Mannheim) und in der Landespolitik (Landtagsabgeordneter und bildungspolitischer Sprecher der Fraktion) sowie seine Tätigkeit als Staatssekretär im baden-württembergischen Kultusministerium verweisen. Ob er als Psychiater ein ganz besonderes Geschick bei der Führung der Verwaltung und bei der Vermittlung zwischen sieben Gruppierungen im Rat hat, in dem es keine klare Mehrheit gibt, bleibt abzuwarten. Jedenfalls scheint seine Karriere im Land schon auf ein außergewöhnlich gutes politisches Gespür zu verweisen. 103

Auch in Frankfurt wurde die Position durch den Abgang der bisherigen beliebten langjährigen Oberbürgermeisterin frei. Es galt fast als sicher, dass der CDU-Kandidat, der Innenminister des Landes Hessen, sie beerben würde. Die Oberbürgermeisterin schlug ihn zudem als Nachfolger vor. Sie hatte sich jahrelang auf eine CDU/Grünen-Koalition im Rat stützen können. Ihr Kandidat brachte von seiner Ausbildung (Rechtsanwalt) und seiner Berufstätigkeit (unter anderem hauptamtlicher Dezernent in Frankfurt) alle Voraussetzungen für das Amt des Oberbürgermeisters mit. Nicht nur seine Tätigkeit in der Frankfurter Verwaltung, sondern auch seine parteipolitische Aktivität zeigte die enge Verbindung zur Stadt. ${ }^{104}$ Außerdem hätte er Erfahrungen aus der Landespolitik einbringen können.

Aber genau diese Verbindungen zur Landespolitik wurden ihm nach allgemeiner Einschätzung zum Problem, denn in Frankfurt schwelte der Konflikt um den Flughafenausbau, vor allem um den Lärm, den die neue Startbahn verursacht. ${ }^{105}$ So konnte der CDU-Kandi-

102 Rüdiger Soldt, Versteher, in: FAZ vom 15. Dezember 2012, S. 12.

103 Vgl. ebenda.

104 Vgl. Lebenslauf von Boris Rhein, http://de.wikipedia.org/wiki/Boris_Rhein (Abruf am 13. Januar 2013).

105 Bei einer Umfrage des Landes Hessen vom Dezember 2011 wurde die Lärmreduzierung von 88 Prozent der Befragten gewünscht, http://www.hessen.de/irj/hessen_Internet?cid=5c9811f0141d 
dat zwar im ersten Wahlgang die meisten Stimmen auf sich vereinigen, in der Stichwahl siegte allerdings sensationell der SPD-Bewerber. Dieser hatte, wie auch sein Gegner, versprochen, alles zu tun, um die Lärmbelästigung zu mildern. Allerdings betonte er, dass er sich im Gegensatz zum CDU-Kandidaten verstärkt um die sozialen Probleme der Stadt (Kinderarmut, bezahlbaren Wohnraum, ältere Menschen) kümmern wolle. ${ }^{106}$

Nach seinem Studienabschluss als Diplom-Politologe lag sein Schwerpunkt in der Sozialpolitik, auch bei seiner Berufstätigkeit in Wohlfahrtsverbänden und ihren Einrichtungen. In der Kommunalpolitik war er stellvertretender Fraktionsvorsitzender der SPD. 107 Seinen Erfolg in der Stichwahl führten die Beobachter auf die Unterstützung durch Wähler der SPD, der Grünen und der Piraten sowie von Flughafengegnern zurück. Der neue Oberbürgermeister selbst sieht ihn auch durch die gelungene Mobilisierung in den Frankfurter Hochhaussiedlungen ermöglicht. Er will sich künftig für eine „konsequent linke Sozialpolitik“ engagieren. ${ }^{108}$ Eine erste Wahlanalyse zeigte, dass die Wahl vor allem durch starke Zugewinne in den „klassischen Grünen-Hochburgen“ gewonnen wurde. Dabei hatten sich drei Grünen-Dezernenten mehr oder weniger offen für den CDU-Kandidaten ausgesprochen. 109 Durch die Wahl des SPD-Kandidaten stand auch die langjährige Zusammenarbeit von CDU und Grünen im Rat der Stadt auf dem Prüfstand. Manche Parteimitglieder der Grünen hatten sich mit dem als rechts geltenden CDU-Kandidaten nicht anfreunden können und ihn nur aus Parteiräson gewählt. Die Basis der Grünen forderte eine ,inhaltliche Erneuerung der Partei“. Die SPD ist nur drittstärkste Fraktion bei insgesamt elf Gruppierungen im Rat.

Während in Frankfurt die Landespolitik mit stadtspezifischen negativen Auswirkungen, aber auch ein eher problematisches Kandidatenangebot der langjährigen Koalition den Wahlsieg des Herausforderers beförderte, so kann in Stuttgart davon ausgegangen werden, dass die von den Grünen angeführte Landesregierung eher positiven Rückenwind für den Kandidaten der Grünen einbrachte. ${ }^{110}$ Erstmals können die Grünen den Oberbürgermeister in einer Landeshauptstadt stellen und die CDU dort in der Spitzenposition nach fast 40 Jahren ablösen. Der von der CDU, der FDP und den Freien Wählern unterstützte parteilose aber CDU-nahe Werbeunternehmer unterlag klar im zweiten Wahlgang.

Während der neue Oberbürgermeister seine parteipolitischen Wurzeln in Stuttgart hat, dann aber seit Jahren zu den führenden Persönlichkeiten der Grünen im Bund gehörte, unter anderem als Parteivorsitzender und Fraktionsvorsitzender im Bundestag (bis 2009), hatte der Gegenkandidat keinerlei praktische politische Erfahrung. Er wollte mit Hilfe einer professionellen Werbestrategie, in die er viel eigenes Geld einbringen konnte ${ }^{111}$, gewinnen und hoffte, dass die bürgerlichen Wähler Baden-Württembergs einen bescheiden auftretenden Selfmademan als ihren Kandidaten schätzen würden. Bei der Nominierung hatte er sich

a8cd4bffa4..., S. 6 (Abruf am 25. Januar 2012). Jochen Remmert / Helmut Schwan, Mit Turbulenzen ist zu rechnen, in: FAZ vom 4. Februar 2012, S. 5.

106 Vgl. Tobias Rösmann, Kampf um den Römer, in: FAZ vom 5. März 2012, S. 3.

107 Vgl. http://www.frankfurt.de/sixcms/detail.php?id=2779\&_ffmpart (Abruf am 13. Januar 2013)

108 http://www.hr-online.de/website/tools/printsite.jsp?key=standard_document_44361349 (Abruf am 13. Januar 2013)

109 Vgl. http://www.hr-online.de/website/tools/printsite.jsp?key=standard_document_44352699 (Abruf am 13. Januar 2013)

110 Vgl. Rüdiger Soldt, Schwäbische Kehrauswoche. Oberbürgermeisterwahl in Stuttgart, in: FAZ vom 19. Oktober 2012, S. 5.

111 Vgl. ebenda. 
mit Zweidrittelmehrheit gegen einen Politiker durchgesetzt, der alle Voraussetzungen für das Amt des Bürgermeisters in Baden-Württemberg mitbrachte. Er hatte Verwaltungswissenschaft in Konstanz studiert, auf verschiedenen Verwaltungsebenen gearbeitet, wurde für zwei Amtszeiten in der Stadt Singen zum Oberbürgermeister gewählt und danach zum Minister für Arbeit und Soziales berufen. Seine Parteikarriere führte ihn bis in den Bundesvorstand der CDU. Die Affären, die ihm öffentlich negativ ausgelegt wurden und zu seinem Rücktritt vom Ministeramt führten, erscheinen Außenstehenden als Petitessen. ${ }^{112}$

Nach dem Abgang des Oberbürgermeisters von Kiel durch seine Wahl zum Ministerpräsidenten in Schleswig-Holstein galt es für die SPD, das wiedergewonnene Oberbürgermeisteramt - zwischenzeitlich gab es für eine Wahlperiode eine CDU-Oberbürgermeisterin in der SPD-Hochburg - zu verteidigen. Die Kandidatin, die präsentiert wurde, entsprach wenig den traditionellen Qualifikationsanforderungen für diese Position: Sie hatte keine Verwaltungserfahrung und war auch als Journalistin bisher Kommentatorin, nicht Gestalterin. Innerparteilich hatte sie sich nur knapp als Kandidatin durchgesetzt, erhielt aber dann von ihrer Partei - und Helmut Schmidt - große Unterstützung im Wahlkampf.

Obwohl die SPD im Rat, dem sieben Gruppierungen angehören, die meisten Sitze hat, ging die Partei also einen risikoreichen Weg. ${ }^{113}$ Dass er zum Erfolg bei der Wahl führte, ist wohl der Kommunikations- und Argumentationsfähigkeit der profilierten Zeit-Journalistin zu verdanken, die ihre Schwerpunkte in der Sozialpolitik setzte (Kinderbetreuung und Wohnungspolitik) und damit bei den Wählern sehr gut ankam. Auch die Unterstützung der Grünen in der Stichwahl war ein wichtiges Element beim Sieg mit 54,1 Prozent. ${ }^{114}$ Der Mangel an Verwaltungserfahrung war aber Ursache für den „erzwungenen“ Rücktritt bereits nach einem Jahr.

Bei der Oberbürgermeisterwahl in Leipzig konnte der bisherige Amtsinhaber 2013 seine Position verteidigen. Die Stadt hatte seit der Wende deutschlandweit bekannte SPD-Repräsentanten als Oberbürgermeister. Dies galt für den bis 2013 gewählten nicht. Er musste sich bei der zum Jahresbeginn 2013 anstehenden Wiederwahl gegen zwei ernstzunehmende Bewerber von CDU und Linken 115 behaupten. Bei den Kommunalwahlen hatte die SPD seit 2004 stark an Zustimmung verloren. ${ }^{116}$ Der SPD-Oberbürgermeister erhielt im ersten Wahlgang mit 40,2 Prozent die meisten Stimmen. Erste Analysen deuteten darauf hin, dass er vor allem von (älteren) Frauen Stimmen erhalten hatte, während die älteren Männer den CDU-Kandidaten, einen ehemaligen Polizeipräsidenten, unterstützten. Bei den Älteren hatte auch die Kandidatin der Linken mehr Anhänger, während der Grünen-Bewerber unter den Jüngeren seine Wähler fand.117

Im zweiten Wahlgang konnte der bisherige Amtsinhaber nicht mit der Unterstützung anderer Parteien rechnen. Mit Ausnahme des FDP-Kandidaten, der im ersten Wahlgang nur

112 Vgl. http://www.kontextwochenzeitung.de/newsartikel.de/newsartikel/2012/03/der-renner-mitdem-guttenberg (Abruf am 14. Januar 2013).

113 Der Gegenkandidat der CDU hatte Verwaltungserfahrung als Kämmerer der Stadt, http://www. ndr.de/regional/schleswig-holstein/kielwahl117.html (Abruf am 12. Februar 2013).

114 Vgl. ebenda.

115 Bundestagsabgeordnete, die bereits 2005 vergeblich antrat und mit Wirtschaftskompetenz wirbt.

116 Vgl. Homepage der Stadt Leipzig, http://www. http://www.leipzig.de/buergerservice-und-verwaltung/wahlen-in-leipzig/stadtratswahlen/ (Abruf am 9. Februar 2013).

117 Vgl. http://www.lvz-online.de/nachrichten/aktuell_themen/obm-wahl-in-leipzig/r-obm-wahl-inleipzig.html (Abruf am 13. Februar 2013) 
1,8 Prozent der Stimmen auf sich vereinigen konnte, kandidierten alle auch im zweiten Wahlgang und sahen Chancen auf den Sieg, was sich in ihrem zum Teil sehr engagierten Wahlkampf zeigte. Der Oberbürgermeister konnte sich aber mit deutlicher Mehrheit (45 Prozent) gegenüber dem ortsfremden und parteilosen CDU-Kandidaten („ein Law und Order-Mann"118) behaupten, der nur 28,7 Prozent erhielt; die Kandidatin der Linken kam auf 15,3 Prozent, der der Grünen auf 9,8 Prozent. Der „Verlegenheitskandidat“ der CDU meinte, dass dem Oberbürgermeister der Amtsbonus zur Wiederwahl verholfen habe. ${ }^{119}$

Auch in Wiesbaden ging es um die Wiederwahl des Amtsinhabers. Der CDU-Oberbürgermeister verdankte - unabhängig von seiner unbestreitbaren fachlichen Kompetenz für das Amt ${ }^{120}$ - seinen überwältigenden Wahlerfolg von 2007 nicht zuletzt der Tatsache, dass ihm ein Gegenkandidat aus der SPD fehlte, weil die Partei ihren Kandidaten nicht rechtzeitig zur Wahl angemeldet hatte. Der 2007 bei nur 26,9 Prozent Wahlbeteiligung mit 65,6 Prozent der Stimmen Gewählte ${ }^{121}$ galt nach seinem deutlichen Vorsprung im ersten Wahlgang 2013 mit 48 Prozent als klarer Favorit bei der Stichwahl. Der Stimmenanteil von 38,4 Prozent seines jungen Herausforderers, des als wenig bekannt geltenden Fraktionsvorsitzenden der SPD, wurde als Achtungserfolg gewertet. Der Oberbürgermeister setzte offenbar ganz auf seinen Amtsbonus; er galt als beliebt, und es gab keine besondere Streitfrage in der Stadt. Er sah seine Ausgangsposition „in der konservativ wirkenden ,Weltkurstadt ${ }^{\prime} \ldots$ als optimal“122. Der Wahlkampfleitung der CDU wurde aber - zumindest in der örtlichen Presse - ein miserables Zeugnis ausgestellt. ${ }^{123}$ Der Herausforderer, Juniorchef einer Druckerei, setzte auf die soziale Komponente, den Zusammenhalt in der Stadt, ein Thema, für das er aufgrund seiner Lebensgeschichte Glaubwürdigkeit einbrachte. ${ }^{124}$ Sein eher modernes Image ${ }^{125}$ machte ihn für jüngere Wähler und Anhänger der Grünen wählbar. Sein engagierter Wahlkampf und die Wahlempfehlung der Kandidatin der Grünen trugen zu seinem knappen Sieg mit einem Vorsprung von 1,6 Prozent der Stimmen bei.

\section{Reformziele erreicht?}

Generell kann festgestellt werden, dass das Wahlverhalten auf der kommunalen Ebene von denselben Faktoren beeinflusst wird wie auf den anderen Ebenen des politischen Systems. Allerdings ist jede Kommune eine spezifische Einheit innerhalb der überörtlichen Systeme. Die wahlrelevanten Faktoren werden von den dort aktiven Organisationen, Personen und Medien in besonderer Weise geprägt. Dabei haben die Parteien nicht nur für die Wahlen auf

118 Ebenda.

119 Vgl. http://www.sz-online.de/sachsen/burkhard-jung-schafft-wiedereinzug-ins-leipziger-rathaus-2511301.html (Abruf am 18. Februar 2013).

$120 \mathrm{Vgl}$. http://www.wiesbaden.de/rathaus/stadtpolitik/oberbuergermeister/portra... (Abruf am 27. Februar 2013).

121 Vgl. Thomas Holl, Bis zur letzten Wählerstimme, in: FAZ vom 23. Februar 2013, S. 4.

122 Ebenda.

123 Vgl. Katja Rietze, OB-Wahl in Wiesbaden: Live-Ticker zur Stichwahl zum Nachlesen, in: Wiesbadener Kurier vom 10. März 2013, http://www.wiesbadener-kurier.de/eilmeldung/ print_12908569.htm (Abruf am 10. März 2013).

124 Vgl. Thomas Holl, Sven Gerich, in: FAZ vom 12. März 2013, S. 8.

$125 \mathrm{Vgl}$. http://spiegel.de/politik/deutschland/wiesbaden-spd-mann-gerich-schlaegt-cdu-oberbuergermeister-mueller-a-887973.html (Abruf am 11. März 2013). 
der kommunalen Ebene, sondern auch während der Wahlperiode keineswegs an Bedeutung verloren. Sie müssen durch ihre politische Arbeit Vertrauen schaffen und erhalten, denn neben den Aktionen des (Ober-)Bürgermeisters in seiner Amtszeit werden die Spitzenrepräsentanten der Parteien - und hier insbesondere die der Ratsfraktionen - durch die Berichterstattung in der Lokalpresse noch am ehesten von der politisch interessierten Öffentlichkeit wahrgenommen. Eine Abfolge von durch die öffentliche Diskussion scharf kritisierten Entscheidungen kann das Image der Kommunalpolitiker stark beeinträchtigen und die Teilnahme an Kommunalwahlen gefährden. Unterschiedliche Niveaus der Wahlbeteiligung bewirken noch immer ebenenspezifisch unterschiedliche Wahlergebnisse für Parlamente und Gemeinderäte. Hinzu kommen die durch Verzicht auf Sperrklauseln erhöhten Aussichten von zusätzlichen Parteien und Gruppen auf den Gewinn von Mandaten in den Kommunen.

Bei der Vorbereitung der Kommunalwahlen haben die Parteien für die direktdemokratische Wahl der (Ober-)Bürgermeister eine hochsensible Entscheidung zu treffen. Dem Kandidaten sollten die Wähler zutrauen, dass er die genügende fachliche Qualifikation für die Führungsposition mitbringt. Allerdings hat es der durch seine bisherige Tätigkeit wenig für die Politik sozialisierte Fachmann schwer, wenn er nicht uneingeschränkt durch die ihn präsentierende Partei unterstützt wird, die eigenen Wähler an die Wahlurne zu bringen. Dies ist besonders dann weniger aussichtsreich, wenn ein neuer Kandidat nur durch eine Kampfkandidatur in seiner eigenen Partei die Nominierung gewonnen hat. Weiterhin ist der Gegenkandidat zu beachten. Wenn der (Ober-)Bürgermeister mit sozialer Kompetenz ausgestattet ist und zur Wiederwahl steht, sich im Amt bewährt hat, also zusätzlich auf den Amtsbonus zählen kann, wird ein Herausforderer selten bei der (Ober-)Bürgermeisterwahl erfolgreich sein. Dann können nur noch Fehler des bisherigen Amtsinhabers (Unterschätzung der Risiken einer demokratischen Wahl oder ein stadtweit wahrgenommenes politisches Streitthema) zugunsten eines Außenseiters eine positive Rolle spielen.

Insgesamt scheint die Direktwahl des obersten Repräsentanten und gleichzeitigen Chefs der Verwaltung die Wahlberechtigen nicht stärker für die Mitwirkung animiert zu haben. Die Wahlbeteiligung bleibt auf einem niedrigen Niveau. Auch die Reformen des Wahlrechts, die den Wahlberechtigten eine Mitentscheidung über die Rangplätze der aufgestellten Kandidaten für den Rat einräumen, haben das Interesse zur Beteiligung an den Kommunalwahlen nicht stärker geweckt. Nur in Bundesländern mit sehr vielen kleinen Gemeinden ist eine höhere Wahlbeteiligung zu registrieren. Aber auch hier wurde die allgemeine Tendenz sinkender Wahlbeteiligung nicht gestoppt. Nach dem Wegfall der Fünf-Prozent-Hürde, die mehr Gruppierungen eine Chance gibt, in den Gemeinderat einzuziehen, zeigt sich bisher ein leichter Zuwachs in der Wahlbeteiligung. Dieser wird allerdings nicht durch völlig neue Parteien verursacht - wie vor allem die Piraten -, sondern durch erkennbar ortsspezifische Gruppierungen, die - wie bisherige Untersuchungen zeigen - häufig Abspaltungen der etablierten Parteien sind.

Insgesamt haben die institutionellen Veränderungen nicht jene Wirkungen gezeigt, die die Reformer erwartet haben: unter anderem eine stärkere Rückbindung der Verwaltungsspitze an die Bürger und weniger Mitwirkung der Parteien an kommunalpolitischen Willensbildungsprozessen. Die Bedeutung der Parteien als wichtige Kommunikations- und Mobilisierungsinstanzen ist nach wie vor beachtlich. Aber sie müssten sich erneuern, das heißt für Mitwirkungsbereite attraktiver und aufnahmefähiger werden, sich als Sozialisationsinstanz für politischen Nachwuchs in der Demokratie verstehen. Die institutionellen Reformen haben dazu aber offenbar keinen Anreiz gegeben. Diese hätte erst dann als eindringliches Signal 
erkannt werden können, wenn ein erheblicher Stimmenverlust unweigerlich mit der Oppositionsrolle für eine Wahlperiode lang verbunden sein würde. Nach Abschaffung der FünfProzent-Hürde können sich Wahlverlierer aber immer noch durch Zusammenarbeit mit Kleinparteien und Gruppierungen die Mehrheit sichern und sich im Nachhinein als Sieger fühlen. Das verschafft denjenigen, die schon lange im politischen Prozess agieren und das politische Handwerkszeug kennen, Vorteile: Sie sind nämlich gefordert, Mehrheitskoalitionen zusammenzubringen und zusammenzuhalten, was sie praktisch über Jahre unersetzlich macht. Der häufig kritisierte Parteienfilz wird so nur noch gefördert. (Ober-)Bürgermeister, die gegen eine solche Mehrheitskoalition, deren Parteien sie bei der Wahl nicht unterstützt haben, ihre Leitungsfunktion in der Stadt wahrnehmen müssen, tun sich schwer; die Effizienz der Verwaltung nimmt Schaden. Gerade die Performanz der lokalen Autoritäten ist aber für die Vertrauensbildung sehr wichtig. ${ }^{126}$ Für die Bürger als Betrachter von außen wird die Zurechenbarkeit von Entscheidungen immer schwieriger. Ihnen mag es nicht mehr wichtig sein zu wählen, weil sich nicht durch Wahlen, sondern erst bei den unvermeidbaren „Koalitionsverhandlungen“, also im Nachhinein, die Machtverhältnisse klären.

126 Vgl. Christian Schnaudt, Politisches Vertrauen, in: Jan van Deth / Markus Tausendpfund, a.a.O. (Fn. 1), S. 297 - 328, S. 324.

\title{
Politische Generationen, demographischer Wandel und Wahlverhalten in der Bundesrepublik Deutschland: Schicksalsjahre des deutschen Parteiensystems?
}

\author{
Carmen Schmidt und Jan Knipperts
}

Die unmittelbaren Nachkriegsjahre waren in Deutschland noch relativ geburtenschwach. Ab Mitte der 1950er Jahre stieg die Geburtenrate dann stark an und erreichte zu Beginn der 1960er Jahre mit über 2,5 Kindern je Frau den höchsten Stand der Nachkriegszeit. Dieser als „Babyboom“ bezeichnete Anstieg endete mit dem so genannten „Pillenknick“ ab Mitte der 1960er Jahre. Seither nimmt die Zahl der Geburten im früheren Bundesgebiet kontinuierlich ab und liegt seit Anfang der 1970er Jahre unterhalb der Sterberate, was in den kommenden fünfzig Jahren zu einem Bevölkerungsrückgang von voraussichtlich 12 bis 17 Millionen führen wird. ${ }^{1}$ Neben einem Bevölkerungsschwund führt diese Entwicklung zu erheblichen Veränderungen in der Altersstruktur: Bereits heute sind 20 Prozent der Deutschen 65 Jahre oder älter, und schon in den kommenden beiden Jahrzehnten wird sich der Anteil älterer Menschen deutlich vergrößern. Weiter verstärkt wird dieser Effekt durch eine stetig steigende Lebenserwartung. ${ }^{2}$

1 Vgl. Hans Peter Friedrich, Die Demographiestrategie der Bundesregierung, in: Michael Hüther I Gerhard Naegele (Hrsg.), Demografiepolitik - Herausforderungen und Handlungsfelder, Wiesbaden 2013, S. 46 - 48, S. 46.

2 Vgl. Elke Drepper-Cramer, Der demographische Wandel in den Kommunen: Antizipatorische politische Entscheidungen angesichts struktureller Umbrüche, Uelvesbüll 2011, S. 25. 